\title{
The Ngai coupling model of relaxation: Generalizations, alternatives, and their use in the analysis of non-Arrhenius conductivity in glassy, fast-ionic materials
}

\author{
J. Ross Macdonald \\ Department of Physics and Astronomy, University of North Carolina, Chapel Hill, \\ North Carolina 27599-3255
}

(Received 1 October 1997; accepted for publication 20 April 1998)

\begin{abstract}
The ionic conductivity of glassy, fast-ion-conducting materials can show non-Arrhenius behavior and approach saturation at sufficiently high temperatures [J. Kincs and S. W. Martin, Phys. Rev. Lett. 76, 20 (1996)]. The Ngai coupling model was soon applied to explain some of these observations [K. L. Ngai and A. K. Rizos, Phys. Rev. Lett. 76, 1296 (1996)], but detailed examination and generalization of the coupling model suggested the consideration of a related, yet different, approach, the cutoff model. Although both the coupling and cutoff models involve a shortest nonzero response time, $\tau_{c}$, and lead to single-relaxation-time Debye response at limiting short times and high frequencies, they involve different physical interpretations of their low- and high-frequency response functions. These differences are discussed; the predictions of both models in the frequency and time domains are compared; and the utility of both models is evaluated for explaining the non-Arrhenius conductivity behavior associated with the dispersed frequency response of $z \mathrm{AgI}+(1-z)\left[0.525 \mathrm{Ag}_{2} \mathrm{~S}+0.475 \mathrm{~B}_{2} \mathrm{~S}_{3}: \mathrm{SiS}_{2}\right]$ glass for $z=0$ and 0.4 . The cutoff approach, using simulation rather than direct data fitting, yielded semiquantitative agreement with the data, but similar analysis using the coupling model led to poor results. The coupling model leads to an appreciable slope discontinuity at the $\tau_{c}$ transition point between its two separate response parts, while the cutoff model shows no such discontinuity because it involves only a single response equation with a smooth transition at $\tau_{c}$ to limiting single-relaxation-time response. The greater simplicity, utility, and generality of the cutoff model suggest that it should be the favored choice for analyzing high-conductivity data exhibiting non-Arrhenius behavior. (C) 1998 American Institute of Physics. [S0021-8979(98)06714-0]
\end{abstract}

\section{INTRODUCTION AND BACKGROUND}

\section{A. General}

In 1996, Kincs and Martin (KM) published an important paper demonstrating very high room-temperature dc conductivity in a series of chemically stable, conductivityoptimized, ion-conducting glasses. ${ }^{1}$ Such high conductivity is very desirable for device applications, but, unfortunately, the full potential of these optimized materials was not realized because of progressive deviation from their lowtemperature Arrhenius behavior toward conductivity saturation, beginning well below their $T_{g}$ values. Thus, for example, instead of reaching the room-temperature extrapolated value of $0.04(\Omega \mathrm{cm})^{-1}$, one of their AgI-doped materials only yielded a value of $0.006(\Omega \mathrm{cm})^{-1}$ at that temperature. Kincs and Martin also suggested that the transition to such non-Arrhenius behavior at higher temperatures is ubiquitous in all superionic fast-ion-conductive glasses.

It thus seems as though Nature somehow acts to restrict the maximum conductivity reachable in the saturated region. In fact, as the present work shows, that conductivity seems to depend only slightly on the degree of doping. Although KM suggested a mobility-oriented qualitative explanation for the approach to saturation, Ngai and Rizos ${ }^{2}$ (NR) soon proposed an alternate and more satisfactory explanation for the devia- tions from Arrhenius behavior, one based on the coupling model $(\mathrm{CM})$ of Ngai and his co-workers. ${ }^{3-38}$ Their results were particularly remarkable because they creatively explained the non-Arrhenius behavior of the dc conductivity by means of a model that involved deviation from a lowfrequency dispersed response only at frequencies of the order of $10^{12} \mathrm{~Hz}$ and above. What an extrapolation!

These works led me to take a closer look at the CM and the basic physics involved in it, with the hope that such an examination might shed further light on those factors leading to saturation in fast ion conductors or, indeed, in any conducting material with a physically necessary crossover at very high frequencies. Identification of the controlling factors might then allow one to optimize such a conductor in order to either entirely avoid saturation or at least to push its onset to higher temperatures.

In the course of my examination of the published work on the CM, I found some limitations in the CM approach, which, in turn, led me to examine a closely related but simpler alternative to the CM, the cutoff model (COM). In the present work, the $\mathrm{CM}$ is corrected and generalized where appropriate; the two models are described in detail; their predictions are compared, in general; and their success in explaining the KM results is evaluated. It should be empha- 
sized that although the results of the application of the Ngai coupling model to the KM data are compared with those of the COM in Sec. II, and although some of the changes in the Ngai CM made by Ngai and his associates over the years are summarized in Sec. I C, the present changes and generalizations of the $\mathrm{CM}$ render it different from the current or earlier versions of the Ngai CM. Thus, in order to maintain the distinction, I shall use, where appropriate, $\mathrm{NgCM}$ to denote the Ngai CM and just CM for the current extensions of the original coupling model or for referring to common features of both approaches.

The coupling model of relaxation was proposed by Ngai in $1979 ; ;^{3-5}$ it has been applied to a wide range of physical phenomena since its inception; and it has been derived by several alternate approaches. Over the years there have probably been well over a thousand pages, published in scientific journals and in the reports of conferences and meetings, that have been devoted to explicating and applying the model. References 2-38 are representative. It is not, however, the large number of published pages devoted to the $\mathrm{NgCM}$ that make it worthy of further consideration, but instead the claims that it can explain a very wide variety of linearresponse, relaxation-related experimental results. For example, Ngai ${ }^{33}$ has said, "The usage of these coupled relations in enhancing the understanding of relaxations in several classes of correlated systems have [sic] been amply demonstrated in the past. Examples of these remarkably successful applications will be given later in the present review. It is worthwhile to emphasize that these coupled relations were derived ... long before they were applied to experiments and subsequently verified by the data."

Most of the great body of work on the $\mathrm{NgCM}$ has directly involved Ngai and his various coauthors, and only a relatively small amount of independent discussion of the approach has appeared in the literature. It is worth mentioning, however, that Ngai rebuts some criticisms of the model at the end of Ref. 15, and replies to comments and questions concerning it in the discussion section appearing at the end of Ref. 23. The discussions in Refs. 19 and 39 are also relevant to the application of the $\mathrm{NgCM}$ to the analysis of the time decay of remanent magnetization. The characteristics of the $\mathrm{NgCM}$ are compared to those of some other relaxation models in Refs. 15 and 22.

As demonstrated below, because of the development of the LEVM computer program for accurate and rapid data analysis appropriate for comparing a fitting model with experimental data, ${ }^{40-42}$ it is now pertinent and possible to examine the content of the $\mathrm{NgCM}$, the methods used in the past to verify it, and finally to evaluate its applicability more quantitatively and accurately than has been possible previously. We begin with a brief summary of the main features of the $\mathrm{NgCM}$, provide a needed generalization of it, and note its connection to the more general COM, one applicable to any relaxation situation. Then the utility of the two models in explaining non-Arrhenius conductivity behavior in glassy fast-ionic conductors is examined. Finally, time and frequency domain responses of the two approaches are compared in detail and discussed. Although the $\mathrm{NgCM}$ has been applied to a wide variety of relaxation processes, ${ }^{2-38}$ includ- ing electrical and mechanical relaxation in disordered materials, for simplicity the present work will be restricted to the small-signal electric response of such materials in the time and frequency domains, the area considered in the original derivation of the $\mathrm{NgCM}^{3-5}$

\section{B. Types of response}

It has recently been shown ${ }^{42-45}$ that in the discussion and analysis of electrical relaxation data it is important to distinguish between dielectric-system dispersive response (DSD) and conductive-system dispersive response (CSD), even though such a distinction is somewhat idealized and fails to capture the full complexity of all but the simplest processes. For DSD, the dispersion is taken to involve such dielectric quantities as rotating or induced dipoles, and the principal dispersion process thus leads to no dc conductivity. It is then most appropriate to describe the data in terms of a model defined at the complex dielectric constant, or susceptibility, level. In contrast, CSD involves mobile charges that are able to contribute to a dc conductivity, $\sigma^{\prime}(0)$, which is an intrinsic part of the dispersive process. Then, a model for such a response is best developed and applied at the complex resistivity or impedance level. Finally, as discussed below, it is important to distinguish between two types of CSD behavior, denoted CSD0 and CSD1. Incidentally, when a DSD situation includes an unrelated, nondispersed dc conductivity, it has been found that its frequency response may be very well fit by either a CSD0 or CSD1 model ${ }^{46}$ (and vice versa), and the limiting $\log -\log$ slopes (termed just slopes hereafter) of DSD and CSD responses are then equal. ${ }^{47} \mathrm{~A}$ choice of the most appropriate model to fit such data is greatly aided when data are available over a range of temperatures.

In terms of a distribution of relaxation times (DRT), $\tau$, DSD can be represented by a Maxwell circuit, ${ }^{48}$ one involving a parallel set of dielectric-entity relaxors, each of which may be formally represented by a resistor and a capacitor in series, in either discrete or differential (continuous) form. These circuit elements model energy dissipation and storage processes. Similarly, the DRT for CSD response can be modeled with a Voigt circuit, ${ }^{48}$ one that involves a series set of resistivity-level relaxors, each of which may be represented by a resistor and capacitor in parallel, in either discrete or differential form. Note that the use of a DRT (or a distribution of activation energies) associated with given temporal or frequency response does not necessarily imply that the theoretical or experimental response is best interpreted physically in terms of such a distribution. But since techniques for distinguishing between discrete and continuous distributions are now available, ${ }^{41,46}$ if one finds that experimental response is best described by one or only a few discrete Debye relaxation times, this will certainly be the appropriate model to describe the physical processes that contribute to the relaxation response.

Let us use the subscript $n$, with $n=D, 0$, or 1 , to designate DSD, CSD0, or CSD1 behavior, respectively. Define $U_{n}$ as an unnormalized measured or model quantity of interest. It is mathematically convenient to express the normalized form of $U_{n}, I_{n}$, in terms of a DRT, say $g_{n}(\tau)$. Let $x$ 
$\equiv \tau / \tau_{k}$, where $\tau_{k}$ is a characteristic response time, and define $y \equiv \ln (x)$. We may then write ${ }^{42-45}$

$$
\begin{aligned}
I_{n}(\Omega) & \equiv \frac{U_{n}(\Omega)-U_{n}(\infty)}{U_{n}(0)-U_{n}(\infty)} \\
& =\int_{0}^{\infty} \frac{G_{n}(x) d x}{(1+i \Omega x)}=\int_{-\infty}^{\infty} \frac{F_{n}(y) d y}{[1+i \Omega \exp (y)]},
\end{aligned}
$$

where $G_{n}(x) \equiv \tau_{k} g_{n}(\tau), F_{n}(y) \equiv x G_{n}(x), \Omega \equiv \omega \tau_{k}$, and the $F_{n}$ form, which may be simply related to a distribution of activation energies, ${ }^{49}$ is particularly appropriate for numerical quadrature. Here the DRTs are normalized, so $I_{n}(0)=1$ and $I_{n}(\infty)=0$, as indicated above.

Consider now some possible connections between the DSD and CSD $I_{n}$ quantities and their associated DRTs. Suppose that the specific form of a DRT, say $G_{D}(x)$, is known for the DSD situation. Then, it has been shown ${ }^{49}$ that this same form may be used in Eq. (1) with $n=D$ or 0 to define either the DSD or CSD0 response, respectively. Here, we shall be primarily concerned with CSD situations, but note that every CSD0 model implies the existence of a DSD one with the same formal DRT. An important quantity common to both CSD and DSD situations is $\epsilon_{D_{\infty}}$, the high-frequencylimiting dielectric constant associated with pure dielectric processes.

Long ago, Macedo, Moynihan, and co-workers ${ }^{50}$ proposed that conductive-system relaxation response could be appropriately expressed at the complex modulus, $M(\omega)$, level in terms of $\tau g_{0}(\tau)$, or, equivalently, $x G_{0}(x)$, thus defining what is here designated as the CSD1 response, a result obtained independently somewhat later. ${ }^{49}$ This later work indicated that CSD1 analysis is more appropriate for conductive-system dispersive situations in which the conductivity is thermally activated than is the CSD0 approach.

The normalized moments of a normalized CSD0 or CSD1 distribution may be expressed as ${ }^{42}$

$$
\left\langle x^{m}\right\rangle_{n} \equiv \int_{0}^{\infty} x^{m} G_{n}(x) d x,
$$

where $^{42-45} G_{1}(x) \equiv\left(x /\langle x\rangle_{0}\right) G_{0}(x)$; and so it follows in this situation that $\left\langle x^{-1}\right\rangle_{1}=1 /\langle x\rangle_{0}$. The connection between the CSD0 and CSD1 response need not be made at the modulus level. It may alternatively be written as ${ }^{42-45}$

$$
I_{1}(\Omega)=\left(\left\langle x^{-1}\right\rangle_{1} / i \Omega\right)\left[1-I_{0}(\Omega)\right] .
$$

It is crucial to recognize that when Eq. (3) is used to fit data, any parameters involved in $I_{0}(\Omega)$, such as a fractional exponent, ones that would ordinarily involve a 0 subscript, must be interpreted as CSD1 quantities and must involve an $n=1$ subscript. Further, although Eq. (3) shows that an expression for $I_{1}(\Omega)$ may be obtained when one for $I_{0}(\Omega)$ is available without explicit knowledge of $G_{0}(x)$ or $G_{1}(x)$ if $\langle x\rangle_{0}$ is known, this is not the case for the transient response. Then, $G_{0}(x)$ is needed to form $G_{1}(x)$ for use in the temporal analog of Eq. (1), that where the quantity $1 /(1+i \Omega x)$ is replaced by ${ }^{42} \exp \left[-\left(t / \tau_{k}\right) / x\right]$.

\section{The coupling model}

The NgCM was originally developed for dielectric polarization involving permanent dipoles or nonpercolating charged particles ${ }^{3,5}$ and thus applied to DSD situations. It has, however, later been used for the analysis of the CSD response as well. ${ }^{2,9,10,14,21,27,30,38}$ The CM involves two separate, coupled response equations; one applying at very short times and high frequencies and the other for long times and low frequencies. A crucial assumption of the CM is that there exists a temperature-independent microscopic time, $t_{c}$, before which the entities that contribute to the relaxation process are uncorrelated, so that interactions do not affect the dynamical relaxation process for shorter times. ${ }^{3-6}$ Let us write $t_{c} \equiv \tau_{c} \equiv 1 / \omega_{c}$, where $\tau_{c}$ has been stated to fall in the range of $10^{-9}-10^{-12} \mathrm{~s}$, with the latter the currently favored value. $^{2,33,34,51}$ For $t>\tau_{c}$, however, the relaxing units begin to become correlated. It is this transition to coupling to a complex environment that has given the $\mathrm{CM}$ its name. In the original $\mathrm{NgCM}$, the complexity of the system is represented by low-lying, correlated-states excitations with a distribution of energy spacings. 5,6

No particular value of the quantity $\tau_{c}$ is predicted by the Ngai theory; instead the presence of $\tau_{c}$ there follows from the assumption of the existence of a cutoff energy of the distribution of low-energy states posited to be present in the material. Similarly, the fractional exponent-type coupling parameter, $n \equiv 1-\beta$, of the theory arises from the assumptions that the density of excitation states at energy $E$ is proportional to $E$ and that $n<1 .{ }^{5,6}$ Although $\mathrm{Ngai}^{17}$ has stated that the $\mathrm{NgCM}$ " does not address the microscopic significance of the parameter $n$,' in later work ${ }^{33}$ he has stated that his Eq. (4.2) of that work, which relates $n$ to an anomalous diffusion coefficient without a detailed analysis, is "the central result of the coupling model.",

Like most present-day relaxation theories that do not involve microscopic interactive many-body analysis, the Ngai approach does not predict explicit temperature dependence for $n$ or $\beta$. Although both the CM and the COM involve by hypothesis the same limiting quantity, $\tau_{c}$, its interpretation is somewhat different for the latter model, as discussed later. The NgCM approach has the virtue that its underlying theory is very general, yielding the possibility of widespread applicability, but the concomitant defect that such generality does not include a detailed description and analysis of specific processes and quantities involved in the actual response of the system. Much the same strengths and weakness are inherent in the COM as well, although it requires fewer assumptions and is more generally applicable than is the CM since it applies to any response model, rather than only to the single specific model of the $\mathrm{NgCM}$. Here we shall omit a further discussion and critique of the physical bases of the $\mathrm{CM}$, already exhaustively covered in the present $\mathrm{NgCM}$ references, and we shall be more concerned with the consequences and structure of the theory than with its metempirics.

As one might expect, there have been some changes in the $\mathrm{NgCM}$ during its 20 years of existence. ${ }^{52}$ Because the changes are instructive and relevant to the present work, and 
because Ngai does not generally relate his changes or corrections to his earlier work, I shall attempt to illuminate some parts of the historical development of the $\mathrm{NgCM}$ and then discuss present extensions to the model.

The $\mathrm{NgCM}$, as it existed in the $1980 \mathrm{~s},{ }^{52}$ was usually defined by the following three time-domain equations: ${ }^{5,6,21}$

$$
\phi_{e}(t)=\exp \left(-t / \tau_{e}\right), \quad t \ll \tau_{c},
$$

the simple exponential response involving the "primitive" relaxation time $\tau_{k}=\tau_{e}$ (termed $\tau_{0}$ by Ngai);

$$
\phi_{s}(t)=\exp \left[-\left(t / \tau_{s}\right)^{\beta}\right], \quad t \gg \tau_{c},
$$

the stretched-exponential [Kohlrausch-Williams-Watts ${ }^{53}$ (KWW)] response for $0<\beta<1$, with a characteristic relaxation time $\tau_{k}=\tau_{s}$; and, finally, an important relation between the $\tau^{\prime}$ s that can be most simply expressed as

$$
\tau_{e} / \tau_{c}=\left(\tau_{s} / \tau_{c}\right)^{\beta} Q,
$$

where, in turn, it is convenient to define

$$
Q \equiv \beta^{-1} \exp \left[-(1-\beta) \gamma_{x}\right],
$$

with $Q=Q_{\mathrm{Ng}}$ when $\gamma_{x}$ has the value appearing in the $\mathrm{NgCM}$. This value was originally ${ }^{4}$ given as 0.57 ; later ${ }^{5,6}$ it was defined as 0.5722; and it was finally ${ }^{9}$ identified as $\gamma$ $\simeq 0.577$, the Euler constant, about 0.57722 , the quantity actually involved in the theory.,

The constant $\gamma_{x}$, not necessarily equal to $\gamma$, is introduced in Eq. (7) to allow $Q$ to vary from its original $\gamma_{x}=\gamma$ theoretical value, which, for $\beta=0.5$, is $Q_{\mathrm{Ng}} \simeq 1.5$. This generalization turns out to be desirable, both because the conditions defining the $\mathrm{NgCM}$ have not remained completely constant since its genesis and because, as demonstrated later for the COM, $Q$ is not temperature independent, even when $\beta$ is. The $\phi(t)$ quantities above are termed relaxation functions for DSD and correlation functions for CSD situations and may also stand for the stress relaxation function for mechanical measurements, etc. ${ }^{13,15,25}$ Although the above expression for $Q_{\mathrm{Ng}}$ appeared in inverse form in the early papers on the $\mathrm{NgCM}$, for several years after 1984, Eq. (7) was simplified ${ }^{16}$ to $Q=1 / \beta=1 /(1-n)$. This change may be interpreted as a rescaling ${ }^{17}$ of $\omega_{c}$, but this was not usually noted, often making the actual definition of $\omega_{c}$ uncertain, as well as making $\omega_{c}$ itself potentially temperature dependent. Finally, the $\ll$ and $\gg$ conditions appearing in Eqs. (4) and (5) have often been replaced, without explanation, by $<$ and $>$ since 1986. $2,23,27,29-34$

In most of the Ngai work, $\beta$ is replaced by $1-n$. When the $\mathrm{NgCM}$ has been applied to CSD0 or DSD situations, $\beta$ has been identified as that associated with stretchedexponential behavior, here denoted KWW0 with $\beta_{0}$ or KWWD with $\beta_{D}$. For CSD1 analysis, however, $\beta=\beta_{1}$, not a pure stretched-exponential parameter, as demonstrated in the next section, although it has usually been so identified. In this case, the relevant KWW response model will be denoted KWW1 with a parameter $\beta_{1}$; Eq. (5) is inapplicable, but a modified CM can still be defined, as discussed later.

Note that when Eq. (7) applies, any temperature dependence of $\beta$ will induce some in $Q$. In the present work, however, I shall just take $Q$ as a proportionality constant whose value is determined by those of the other quantities in Eq. (6). In the modern version of the $\mathrm{NgCM}^{52}$ applying since the early 1990's, however, $Q$ is given the value of unity, one that follows from setting $\phi_{e}\left(t_{c}\right)=\phi_{s}\left(t_{c}\right)$. Deviations from this value are a measure of the appropriateness of this choice. In particular, for a KWW1 CM or COM response, since Eq. (5) does not apply, $Q \neq 1$.

In the present work, we shall be primarily concerned with KWW0 and KWW1 temporal and frequency response for three situations: no cutoff (NCO), the COM, and the CM. Since the NgCM deals only with the KWW0 (or KWWD) temporal response of Eq. (5), the scope of the present analysis and results is far greater than that of that approach. The distinction between $\beta_{0}$ and $\beta_{1}$ is considered in more detail in Refs. 45 and 46, and in Ref. 45 it is also shown that the neglect of the DSD quantity $\epsilon_{D \infty}$ in the usual Moynihan modulus fitting approach ${ }^{50}$ can render the resulting estimates of $\beta$ quite inaccurate and misleading.

The $\gg$ restriction of Eq. (5) appears in the original work on the $\mathrm{NgCM}^{4-6}$ and is necessary to indicate that the KWWrelated expression found in the analysis is only asymptotic. Furthermore, although in most of the published work on the $\mathrm{NgCM}$, the fractional-exponential Eq. (5) expression is taken as the first, ${ }^{33}$ or first "universal" relation ${ }^{16}$ of the $\mathrm{NgCM}$, the full analysis actually yielded ${ }^{5,6}$ an expression only for $d \phi(t) / d t$ in the region $t \gg \tau_{c}$. If that expression is compared with the derivative of Eq. (5), one finds that, except for a scale factor involving the dielectric polarization strength, an additional $\left(\tau_{s} / \tau_{c}\right)^{1-\beta}$ term is present in the original, a term that should then presumably multiply Eq. (5) in order to make it consistent with the original $\mathrm{NgCM}$ theory. Unfortunately, the calculation of $\phi(t)$ itself from the original analysis over the entire time range of interest would require double numerical integrations of an exponential of a cosineintegral function, sufficiently complex and prone to error that no such results have been published.

Although the important Equation (6) follows from the $d \phi(t) / d t$ expression in the original Ngai analysis (when $Q=Q_{\mathrm{Ng}}$ ) and was termed the second universality ${ }^{12,16}$ of the $\mathrm{NgCM}$, if one sets $\phi_{e}\left(\tau_{c}\right)=\phi_{s}\left(\tau_{c}\right)$, the result is just Eq. (6) with $Q=1$, not the original $Q_{\mathrm{Ng}}$ value. To obtain this result, it is necessary that the $\ll$ and $\gg$ conditions of Eqs. (4) and (5) be replaced by $\leqslant$ and $\geqslant$. Ngai and Rizos have implicitly done so in the work of Ref. 2, where they present $\phi(t)$ curves for the full range of $t$, including the crossover point $t=\tau_{c}$. But the choice $Q=1$ is inconsistent with the original theory in the asymptotic limit, and it is unlikely that that theory would lead to $Q=1$ for $\beta<1$ at the crossover point were it practical to calculate its predictions there.

Rendell and Ngai ${ }^{16}$ have stated, "The first and second universalities together address many physical questions concerning the meaning of relaxation and its relation to the material structure," and "a true activated process is not required for the second universality," but, of course, the $\mathrm{NgCM}$ has been often applied to thermally activated CSD situations. Finally, Ngai and Rendell ${ }^{25}$ have stated, "Although $n$ may be a function of temperature, the relation between the effective KWW relaxation time $\tau_{s}$ and the microscopic $\tau_{e}$ [here Eq. (6)] continues to hold at all 
temperatures.' In fact, as shown herein, even for a temperature-independent value of $n$, Eq. (6) only holds for the COM approach (or a CM fit to it) if $Q$ is allowed to be strongly temperature dependent at high temperatures, inconsistent with the original $\mathrm{NgCM}$ second-universality relation.

For completeness, it is worth mentioning that Ngai and his collaborators ${ }^{27,33}$ have presented a simplified version of the $\mathrm{NgCM}$ theory, which involves a constant reaction rate of $\tau_{e}^{-1}$ for $t<\tau_{c}$ and a time-dependent, inverse-power-law, reaction rate involving $n$ for $t>\tau_{c}$. Integration then leads to Eq. (4) with $<$ instead of $\ll$, Eq. (5) with $>$ instead of $\gg$, and Eq. (6) with $Q=1 / \beta$, inconsistent with matching at $t$ $=\tau_{c}$, but characterized as a minor inconsistency by Ngai, ${ }^{52}$ one that could be overtly eliminated by the renormalization of $\tau_{c}$. Incidentally, in this piecewise approach, the presence of $\tau_{e}$ in Eq. (6) arises because of the assumption that the time-varying reaction rate is proportional to $\tau_{e}^{-1}$. Ngai and Rendell $^{25}$ have stated that the basic physics of the NgCM lies in the relaxation-rate equations rather than in the $\mathrm{KWW}$ function. Finally, it is worth noting that in Ref. 34, published in 1995, it was stated that the three coupled equations of the $\mathrm{NgCM}$ (written there with no $Q$ term present, equivalent to $Q=1$ ), "were first proposed more than 14 years ago and have remained unmodified.",

Suppose that one continued to require that Eq. (6) should hold and maintained continuity at $t=\tau_{c}$, even for $Q$ unequal to unity? On multiplying Eq. (5) by a consistency factor $A$ and setting the equations equal at $t=\tau_{c}$, one then finds that Eq. (5) should be replaced by

$$
\begin{aligned}
\phi_{s}(t) & =\exp \left[(Q-1) /\left(\tau_{e} / \tau_{c}\right)\right] \exp \left[-\left(t / \tau_{s}\right)^{\beta}\right] \\
& =\exp \left\{\left[\left(\tau_{c} / \tau_{s}\right)^{\beta}-\left(t / \tau_{s}\right)^{\beta}\right]-\left(\tau_{c} / \tau_{e}\right)\right\}, \quad t \geqslant \tau_{c},
\end{aligned}
$$

and Eq. (4) by

$$
\phi_{e}(t)=\exp \left(-t / \tau_{e}\right), \quad t \leqslant \tau_{c} .
$$

Clearly the prefactor in Eq. (8) is quite different from $\left(\tau_{s} / \tau_{c}\right)^{1-\beta}$, and it reduces to unity when $Q=1$. Further, it approaches unity at low temperatures as $\tau_{e} / \tau_{c}$ increases. In the absence of analytical or numerical results from the original Ngai analysis in the neighborhood of $t \geqslant \tau_{c}$, Eqs. (6), (8), and (9) represent a consistent KWWD or KWW0 coupling model, although one not in full agreement with the original NgCM analysis. ${ }^{4-6}$ Although Eqs. (8) and (9) lead to the same result at $t=\tau_{c}$, it is clear that there will be a discontinuity in slope at this point. As Ngai, Rajagopal, and Teitler ${ }^{20}$ have pointed out, the discontinuity in the $\mathrm{NgCM}$ is an artifact of the piecewise construction of the relaxation function and "a completely satisfactory treatment would presumably be continuous in all derivatives.' The cutoff model, which does lead to the response that is continuous for all derivatives, and to Eq. (6) for both temporal and frequency response, will be discussed in the next section.

Both the CM and the COM lead to an important result for thermally activated systems that involve relaxation-time Arrhenius behavior. Suppose that $\tau_{s}(T)=\tau_{s \infty} \exp \left(E_{s} / k T\right)$, where $\tau_{s \infty}^{-1}$ is an attempt frequency and $E_{s}$ is, as usual, also taken to be temperature independent. Then write $\tau_{e}(T)$ $=\tau_{e^{\infty}} \exp \left(E_{e}(T) / k T\right)$ and substitute these results into Eq. (6) with $\tau_{c}$ and $\tau_{e \infty}$, taken independent of temperature. The result may be put in the form

$$
E_{e}(T)=E_{e 0}+k T \ln [Q(T) / Q(0)],
$$

where $E_{e 0}$ and $Q(0)$ are low-temperature-limiting values for the relaxation process considered, and $E_{e 0} \equiv \beta E_{s}$. From the analysis of a fast-ion conductor presented in Secs. II and III, one finds that $Q$ remains close to $Q(0)$ over an appreciable temperature range, and in this low-temperature range the original $\mathrm{NgCM}$ result, ${ }^{9} E_{e}=E_{e 0}=\beta E_{s}$, with $E_{e}$ assumed to be temperature independent, will be a good approximation. But unless $Q(T)=1$ for all temperatures, the modern form of the $\mathrm{NgCM}$ is not applicable.

Ngai identifies the observed macroscopic activation energy, $E_{s}$, as not fundamental but derivative and instead takes $E_{e 0}$ as the basic microscopic activation energy. ${ }^{5,9,16}$ Incidentally, since it is rare for a DSD, $\tau_{k} \equiv \tau_{D}$, to exhibit Arrhenius behavior over a wide temperature range, ${ }^{54}$ and the CSD1 approach is much more appropriate for thermally activated conductive-system situations than is the CSD0 one, Eq. (10) should apply primarily for CSD1 situations. Although Rajagopal and Ngai ${ }^{15}$ have stated that most relaxation theories, including DRT ones, do not lead to the second universality relation of the $\mathrm{NgCM}$, Eq. (6), with $Q=Q_{\mathrm{Ng}}$ (or currently, $Q=1$ ), the present more general Eq. (6) with temperaturedependent $Q$ is likely to be applicable for any physically realizable relaxation model with power-law behavior at high frequencies before cutoff effects dominate.

Excellent agreement with the $E_{e 0}=\beta E_{s}$ relation has been found by Ngai and his collaborators ${ }^{9,27,28}$ using nuclear spin lattice relaxation results to obtain $E_{e}$ estimates and employing conductivity relaxation results to obtain estimates of $\beta$ and $E_{s}$. There are, however, some potential problems with these results. First, as already mentioned, there are likely to be appreciable inaccuracies in estimates of $\beta$ obtained with the common conductivity-relaxation-analysis approach used in the past, the Moynihan modulus formalism, ${ }^{50}$ as discussed recently. ${ }^{42,45,47}$ Second, when a KWW0 response model is used for the CSD0 response, the related CSD1 transient response is not of the Eq. (5) stretched exponential form, and, concomitantly, the CSD1 KWW1 frequency response is not the same as the CSD0 KWW0 frequency response, as discussed later herein. It is worthwhile to demonstrate the departure from stretched exponential behavior for both the KWW0 and KWW1 response models, and such results appear in the next section. The above possible problems suggest that further tests of the present activation-energy relations might prove useful, although it is shown herein that the $E_{e 0}=\beta E_{s}$ relation applies well for the KM COM analysis situation below temperatures where appreciable conductivity saturation occurs.

Because the KWW1 temporal response is not of the stretched-exponential character of Eqs. (5) or (8), it should be clear that the $\mathrm{NgCM}$, defined in the time domain, is only fully applicable for the KWWD and KWWO temporal response. If we match exponential and KWW1 time response or Debye and KWW1 frequency response at $t=\tau_{c}$ or $\omega$ $=\omega_{c}$, we expect that although such equations as (6), (7), and 
(10) should still apply, they will involve different $Q$ values than those appropriate for KWWD and KWW0 situations. Unfortunately, these distinctions have not usually been recognized in the past when applying the $\mathrm{NgCM}$ to CSD situations, and KWW0 analysis has been used for situations where the KWW1 approach seems to be more appropriate.

\section{The cutoff model}

Although many empirical or semiempirical frequencyresponse models that are associated with a continuous DRT, such as those of Davidson and Cole ${ }^{55}$ and the stretched exponential, ${ }^{53}$ involve a physically realizable low-frequency response, their high-frequency behavior is not necessarily limited by a smallest nonzero response time. For example, both the Davidson and Cole and KWW models lead to a high-frequency-limiting power-law frequency response involving $\sigma^{\prime} \propto \omega^{\xi}$, where $0<\xi<1$. Thus, the $\omega \rightarrow \infty$ limit of the corresponding conductivities is infinite. Physical realizability requires, however, that any response must involve a shortest nonzero response time, here defined as $\tau_{c}$, and a longest noninfinite one. ${ }^{56} \mathrm{~A}$ hierarchy of processes is present in any given real material, and each process will involve such limits. Here we consider only those limiting response times appropriate for small-signal electrical relaxation/ dispersion and omit such contributors to the response as phonons. $^{51}$

The problem of the nonphysical limiting high-frequency or short-time response is sidestepped in the CM by its assumption of an abrupt transition from KWW behavior to the simple exponential response at $\tau \leqslant \tau_{c}$. The COM achieves the same result in a smoother and simpler fashion by cutting off a DRT (or distribution of activation energies) at some nonzero small value of $\tau, \tau_{\min }$, which is here equated to $\tau_{c}$. Such a cutoff yields a smooth transition from the dispersive response, say that of a KWW form, to nondispersive limiting Debye relaxation in the frequency domain or to a simple exponential response in the time domain. Cutoff is by no means a new approach, and it much antedates the initial $\mathrm{NgCM}$ work. In fact, cutoff DRTs have been used since, and before, Frochlich's ${ }^{57} 1949$ discussion of the non-Debye dielectric response associated with a rectangular box distribution. A modern application, involving an effective-medium approach with cutoffs of an initial activation-energy box distribution, appears in Ref. 58.

It is worth emphasizing that it is unnecessary, although often convenient, to define cutoff in terms of a DRT. Any physically realizable response model, such as the correlated hopping (jump) model of Funke, ${ }^{59}$ must show cutoff effects because of the above restrictions on the range of possible response of a given relaxation process. In the frequency domain, these effects lead, in a complex plane plot of, say, the complex resistivity, to vertical approaches of the imaginary part of the resistivity to the real resistivity axis at the extremes of frequency and to a high-frequency plateau in the real part of the conductivity. Funke, Cramer, and their associates $^{51}$ have recently measured the frequency response of an ion-conducting glass up to about $50 \mathrm{THz}$ and find, after vibrational effects are removed, that the hopping conductiv- ity indeed reaches a plateau at a roughly temperatureindependent frequency of about $1 \mathrm{THz}$, consistent with the choice $\tau_{c}=10^{-12} \mathrm{~s}$.

In Ref. 59, Funke compares the original $\mathrm{NgCM}$ to his jump relaxation model and finds both similarities and differences. Although the jump relaxation model also exhibits crossover between types of response below and above $\tau_{c}$, it avoids the slope discontinuities present in the CM. The interesting question remains as to whether the COM can not only simulate jump-relaxation response accurately but can do it in a simpler way for a wider range of experimental response than possible with the more specific and much more complicated jump approach.

If one has available a $G(x)$ expression defined over the full range of $x=\tau / \tau_{k}$, the present type of cutoff is achieved by replacing one or more of the integration limits of Eq. (1) by finite, nonzero values. Because the temporal and frequency response then follow through integration, a kind of averaging process, such sharp cutoffs of the distribution do not produce the slope discontinuities inherent in the CM. For numerical work, it is unnecessary to use an infinite or exceedingly large value for the upper integration limit because of the large- $\tau$ cutoffs inherent in the form of the KWW and Davidson-Cole models. In terms of the $y$ variable, it has been found adequate to use a maximum upper limit of $y$ $=y_{\max } \geqslant 15$ or so. The lower limit of the rightmost integral of Eq. (1) with cutoff becomes just $y_{\min } \equiv y_{c} \equiv \ln \left(\tau_{c} / \tau_{k}\right) \equiv \ln \left(x_{c}\right)$, with $\tau_{k}=\tau_{s}$ for the KWW response. Note that except at high temperatures $y_{c}$ will usually be negative. Further, a value of -60 or less for $y_{c}$ yields no cutoff effects in any measurable frequency range. At a sufficiently high temperature, however, $y_{c}$ will approach the upper limit of the integral, yielding a very nearly single-relaxation-time response over the full time or frequency domain. Incidentally, the effects of different cutoff values for an exponential distribution of relaxation times have been illustrated long ago for both the time $^{60}$ and frequency domains. ${ }^{49}$

In the present work, we shall illustrate the COM response for various KWW situations. To do so, one needs to be able to calculate $G_{n}(x)$ or, equivalently, $F_{n}(y)$, with and without cutoff. For the KWW0 situation with $\beta_{0}=0.5$, the latter quantity, without cutoff, is given by ${ }^{42}$

$$
F_{0}(y)=(1 / 2 \sqrt{\pi}) \exp [0.5 y-\exp (y) / 4] .
$$

No general expression for $F_{0}(y)$ is known for arbitrary $\beta_{0}$, making it difficult but not impossible to calculate the KWW0 and KWW1 frequency response accurately for an arbitrary $\beta$ value. ${ }^{40,42,45}$ In fact, the current V. 7.01 of the LEVM fitting program allows such a frequency response to be calculated extremely accurately and used for fitting when cutoff plays no role. ${ }^{40}$ In addition, the soon-forthcoming version of LEVM, V. 7.1, includes an algorithm for the accurate calculation of the KWW temporal or frequency response with an arbitrary amount of cutoff and arbitrary $\beta$, needed to allow fitting the COM to appropriate data. The present work, however, deals principally with the $\beta=0.5$ choice since closed-form expressions for $G_{0}(x)$ and its moments for $\beta_{0}=0.5$ are available and have been presented previously for arbitrary $x_{c}$ cutoff values. $^{42}$ 


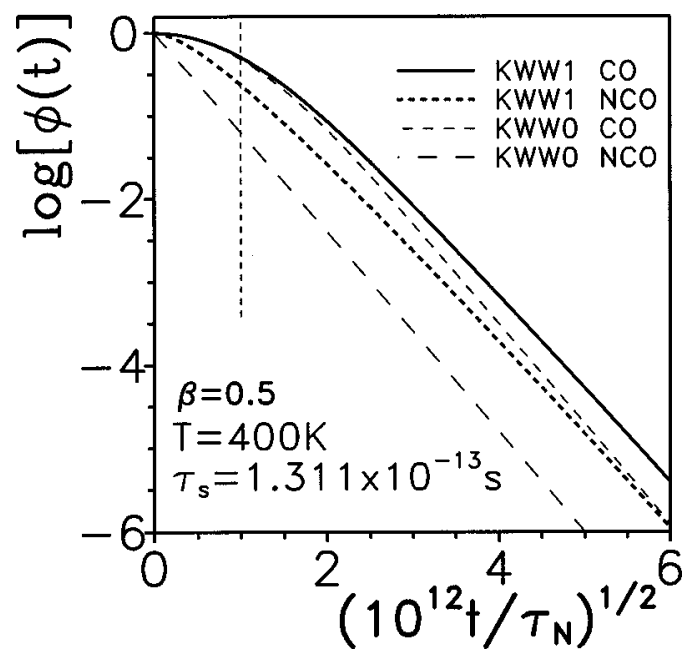

FIG. 1. Some temporal-response relaxation curves for KWW1 and KWW0 models with (CO) and without (NCO) small- $\tau$ DRT cutoff at $\tau=\tau_{c}$. Here $\beta_{1}=\beta_{0}=0.5, \tau_{c}=10^{-12} \mathrm{~s}$, and $\tau_{N}=1 \mathrm{~s}$ here and hereafter. The $x$ axis involves $t^{0.5}$ in order to show the approach to stretched-exponential behavior. The $\tau_{s}$ value is that appropriate for the $T=400 \mathrm{~K}, z=0$ ionic-conductor material analyzed in Sec. II.

Some characteristic temporal responses are illustrated in Fig. 1. These essentially exact results have been calculated using the appropriate expressions for $F_{n}(y)$. Here we contrast KWW1 and KWW0 responses, each with cutoff, thus yielding COM behavior, and response without it. The abscissa is taken proportional to $t^{1 / 2}$ in order to immediately show similarities and differences from an ordinary stretched exponential response with $\beta_{0}=0.5$. The vertical dashed line indicates the position of $\tau_{c}$. The value of $\tau_{s}$ used here is that following from the analysis of the next section for $T$ $=400 \mathrm{~K}$. In addition, that analysis yielded a value of the cutoff parameter $y_{c}$ of 2.032 for this relatively high temperature. All these values are used for both the KWW1 and KWW0 responses.

Figure 1 shows that the KWW0 response without cutoff is just stretched exponential behavior, as required from the present definitions. But the figure also demonstrates that only for $t \gg \tau_{c}$ do the other curves appear to approach the stretched-exponential response. Note that the limiting slopes of the two cutoff lines are the same, as are those without cutoff. At the present temperature, we see that the KWW1 curve is not of the stretched-exponential form over its full range, even in the absence of cutoff. But the present type of presentation is not very sensitive to departures from the stretched-exponential response, and the more detailed analysis presented in Sec. III B indicates that true stretchedexponential behavior only appears for the KWW0 without cutoff; all other situations lead to the stretched-exponential response with time-dependent $\beta$, indicating that Eq. (5) is then not appropriate, except perhaps over a very limited temporal range.

Although one does not need to know $\tau_{e}$ in order to calculate the COM temporal response, its value may be estimated by fitting the response over a time range where simple exponential behavior, arising from cutoff, is dominant. For the present value of $\tau_{c}$, such a fitting has been carried out over the range $10^{-16} \leqslant t \leqslant 10^{-14} \mathrm{~s}$. Similarly, when frequency-response data are available, one can fit the Debye range of $10^{14} \leqslant \omega \leqslant 10^{16} \mathrm{r} / \mathrm{s}$ in order to estimate $\tau_{e}$. For cutoff time and frequency response data calculated as described above, one obtains for $T=400 \mathrm{~K}$ the following $\tau_{e} / \tau_{c}$ estimates for frequency and time, respectively, KWW1: 1.35 , 1.46, and KWW0: 1.28, 1.35. These values lead, using Eq. (6), to $Q$ estimates of the order of 4, very different from 1 . There is, of course, no single-relaxation-time response region for the NCO KWW0 temporal data, but fitting of the NCO KWW1 response with the stretched-exponential model over the above small- $\tau$ range yields $\tau_{e} / \tau_{c}=0.373$ and $\beta_{0}$ $=0.948$, not the simple-exponential response but close to it. More $Q$ results are presented in Sec. III.

It is worth emphasizing that for real data of a sufficient range, LEVM fitting with a response model such as that of Davidson and Cole, for which $G(x)$ is known (or can be rapidly and accurately calculated numerically, e.g., the KWW) for all values of its power-law exponent, $\beta$, leads not only to estimates of $\tau_{s}$ and $\beta$ but to one of $y_{c}$ as well, yielding an estimate of $\tau_{c}$. As shown in the present work, because of the saturation effect associated with a nonzero value of $\tau_{c}$, one need not measure the frequency response up to frequencies of the order of $\omega_{c} \equiv \tau_{c}^{-1}$; instead, it is only necessary to have data available over a limited frequency range at a temperature where some $\tau_{c}$-related deviation from dcconductivity Arrhenius behavior is present.

\section{EXPLANATION OF NON-ARRHENIUS BEHAVIOR IN FAST IONIC CONDUCTORS}

As mentioned in the Introduction, Ngai and Rizos ${ }^{2}$ have recently claimed that the $\mathrm{NgCM}$ can quantitatively explain the non-Arrhenius conductivity of glassy, fast-ionic conductors, and they have demonstrated their approach using the $z$ $=0$ results of Kincs and Martin ${ }^{1}$ for the $z \mathrm{Ag} \mathrm{I}+(1-z)$ $\times\left(0.525 \mathrm{Ag}_{2} \mathrm{~S}+0.475 \mathrm{~B}_{2} \mathrm{~S}_{3}: \mathrm{SiS}_{2}\right)$ glass, data that NR "chose to model as closely as possible." Although the pioneering NR work is a simulation/modeling of the situation and does not involve direct fitting of the actual KM $z=0$ data, NR make the remarkable statements that, "The non-Arrhenius temperature dependence of the experimental data of $\mathrm{KM}$ is reproduced in its entirety by the calculations using the coupling model without any unknown or indeterminable parameter," and, “...the coupling scheme can reproduce this nonArrhenius temperature dependence quantitatively without the introduction of any unknown or indeterminable parameter." Here, it will be shown that, even though the NR results predict an approach to conductivity saturation at high temperatures, they are not in adequate qualitative agreement with the data of KM, and it will be demonstrated how the COM approach may be used to achieve semi-quantitative agreement, as well as an additional physical insight into the phenomena present.

First, let us list the actual parameters and their values used in the NR analysis and then demonstrate how that analysis may be modified and corrected. Note that in a simulation, no model parameter values are determined by direct fitting of the model to the data, and it is thus necessary to 
rely on other information to obtain what one hopes will be an appropriate set. But NR claim that the $\mathrm{NgCM}$ they use for their analysis is "parameterless," suggesting that no parameters are present and thus no values are needed. This is not the case. The following values were actually used by NR, apparently based on guesses and/or values found in other experiments for other materials: $\beta_{0}=0.4, \tau_{c}=10^{-12} \mathrm{~s}$, and $\epsilon_{\infty}=15$. In addition, NR used the $z=0 \mathrm{KM}$ low-temperaturelimiting activation energy estimate, $E_{s}$, of $0.33 \mathrm{eV}$ and the KM $T=298 \mathrm{~K}$ low-temperature-extrapolated dc conductivity, $\sigma_{\mathrm{dc}} \equiv 1 / \rho_{s}=0.0014(\Omega \mathrm{cm})^{-1}$. Finally, the glassy fastionic material analyzed here and by NR is a thermally activated conductive system, and so should be analyzed by a CSD1 approach, as done using the KWW1 below. But in the work of Ref. 2, NR show only the KWW0 results and apparently did not recognize that their use of the Moynihan CSD1 formalism should require that the stretched-exponential behavior of their Eq. (3) [the present Eq. (5) with $t \geqslant \tau_{c}$ ] should be replaced by the KWW1 temporal response, the response not fully of stretched-exponential character.

Since Arrhenius behavior was observed by KM at sufficiently low temperatures, the above $\sigma_{\mathrm{dc}}$ value may be used to obtain the temperature dependence of the dc resistivity, $\rho_{s}$, in the low-temperature region only,

$$
\begin{aligned}
\rho_{s}(T) & =\rho_{s \infty} \exp \left(E_{s} / k T\right) \\
& =0.0018748 \exp (0.33 \mathrm{eV} / k T) \quad(\Omega \mathrm{cm}),
\end{aligned}
$$

in agreement with the NR result. Ngai and Rizos then used the electric modulus formalism ${ }^{50}$ in order to obtain an expression for the temperature dependence of the $\tau_{s}$ of Eq. (5). Although NR characterized this approach as "formal," since the actual DRT introduced in this formalism may be of only mathematical use and not necessarily of physical significance, it is interesting that in earlier work Ngai and coauthors ${ }^{61}$ invoked the fundamental Paley-Wiener Fouriertransform criterion to show that simple exponential decay (and thus a continuous or discrete distribution of single relaxation times such as that considered here) is not a viable description of relaxation phenomena. Luckily for DRT analysis and for the $\mathrm{CM}$, it has recently been shown that the Paley-Wiener criterion does not, in fact, preclude simple exponential relaxation response. ${ }^{62}$

The pertinent equation following from the Moynihan modulus formalism may be written as ${ }^{50}$

$$
\sigma_{\mathrm{dc}}(T)=\epsilon_{V} \epsilon_{\infty} /\langle\tau\rangle_{0} \equiv \epsilon_{V} \epsilon_{\infty} /\left[\tau_{s}(T)\langle x\rangle_{0}\right],
$$

where $\epsilon_{V}$ is the permittivity of vacuum, and in the original work $\epsilon_{D \infty}$ appeared in place of $\epsilon_{\infty}$. In spite of the widespread usage of Eq. (13) since 1973, it has recently been shown to be incorrect. ${ }^{42,45,47}$

For CSD situations, in addition to the ubiquitous $\epsilon_{D^{\infty}}$, there is an additional frequency-dependent effective real dielectric constant present, ${ }^{42} \epsilon_{C}(\omega)$. Its low- and highfrequency-limiting values are denoted as $\epsilon_{C 0}$ and $\epsilon_{C \infty}$ and are purely conductive-system quantities. The full highfrequency-limiting dielectric constant is thus $\epsilon_{\infty}=\epsilon_{C \infty}$ $+\epsilon_{D \infty}$. In many cases of interest, $\epsilon_{C \infty}\left(<\epsilon_{C 0}\right)$ is not negligible in size compared to $\epsilon_{D \infty}$. Unfortunately, the distinction between $\epsilon_{C \infty}$ and $\epsilon_{\infty}$ has usually gone unrecognized in the past, and fitting measurements have yielded an estimate only of $\epsilon_{\infty}$, rather than of both $\epsilon_{C_{\infty}}$ and $\epsilon_{D \infty}$ separately. A recent LEVM analysis of $\mathrm{Na}_{2} \mathrm{O}-\mathrm{SiO}_{2}$ at $321 \mathrm{~K}$ yielded, ${ }^{47}$ however, estimates of these quantities of about 5.3 and 4.8, respectively, consistent with the $\epsilon_{\infty}$ value of the order of 10 often quoted for such glassy materials. Further, an earlier analysis ${ }^{44}$ of $\mathrm{CaTiO}_{3}: 30 \% \mathrm{Al}^{3+}$ suggested that $\epsilon_{C 0}$ was about 60 at $422 \mathrm{~K}$, certainly quite different from any likely $\epsilon_{D \infty}$ value.

With all possible temperature dependencies shown explicitly, the correct equation for the CSD1 response, one that replaces Eq. (13), is ${ }^{42,45}$

$$
\rho_{s}(T)=\tau_{s}(T) /\left[\epsilon_{V} \epsilon_{C \infty}(T)\left\langle\{x(T)\}^{-1}\right\rangle_{1}\right],
$$

which can be rewritten with temperature dependence suppressed as

$$
\rho_{s}=\tau_{s}\langle x\rangle_{0} /\left(\epsilon_{V} \epsilon_{C \infty}\right) .
$$

Note that even when $\epsilon_{C \infty}$ is less than unity, as it is for the present $\mathrm{KM}$ data, it plays a crucial role in determining the ratio $\rho_{s} / \tau_{s}$.

Here, Eq. (15), unlike Eq. (13), involves only conductive-system quantities, as it should. Note that although $\left\langle x^{-1}\right\rangle_{1}$, and thus $\langle x\rangle_{0}$, do not involve $\tau_{s}(T)$ directly, they will, in general, involve possibly temperature-dependent shape parameters, such as the $\beta$ of Eq. (5), and they may be strongly affected by the presence of cutoff of the DRT involved in a fitting model. Further, as already mentioned, if a KWW1 fit of frequency-response data yields an estimate of $\beta$, it should be designated $\beta_{1}$, then used in $\left\langle x^{-1}\right\rangle_{1}$ or $\langle x\rangle_{0}$, and distinguished from the different quantity $\beta_{0}$, which is approximately given by ${ }^{45-47} 1-\beta_{1}$. For real data situations, ones where $\epsilon_{D \infty}>1$, it should also be emphasized that since the standard methods of Moynihan modulus-formalism fitting do not take separate account of the effect of $\epsilon_{D_{\infty}}$, a quantity always present, the $\beta$ obtained from such a fitting, not distinguished from $\beta_{0}$ in the past, is usually a poor estimate of $\beta_{1}$.

For a KWWD or KWW0 response, the general expression for $\langle x\rangle_{0}$ is ${ }^{42,50}$

$$
\langle x\rangle_{0}=\Gamma(1 / \beta) / \beta,
$$

where $\Gamma$ is the ordinary gamma function, and $\beta$ is $\beta_{0}$ or $\beta_{D}$, respectively. But, as discussed above, for the CSD1 response, it is $\beta_{1}$. Equation (16) only applies exactly in the absence of cutoff. Therefore, it is not appropriate to employ it, except in the low-temperature region, where the highfrequency simple-Debye part of the response at frequencies in the neighborhood of $\omega_{c}$ and larger is a completely negligible part of the full response. At higher temperatures, for both the $\mathrm{CM}$ and the COM one should use the cutoff version of Eq. (16) for the KWW0 response. As shown earlier, ${ }^{42}$ the appropriate expression for $\beta=0.5$ is

$$
\langle x\rangle_{0}=4 \Gamma\left(\frac{3}{2}, x_{c} / 4\right) / \Gamma\left(\frac{1}{2}, x_{c} / 4\right),
$$

where $\Gamma(a, z)$ is the incomplete gamma function. $\langle x\rangle_{0}$ decreases as $x_{c}$ decreases and reaches a value of 2 when $x_{c}=0$, as does Eq. (16) for $\beta=0.5$. 
Ngai and Rizos did not use Eq. (17) in their work. But, recognizing that Eq. (16) was inappropriate, they obtained approximate values of $\langle x\rangle_{0}$ at several temperatures above $275 \mathrm{~K}$ by using Eq. (2) with approximate estimates of $G_{0}(x)$. The latter quantities were themselves obtained by a Tikhonov inversion of the full $\mathrm{NgCM}$ temporal response, the combination of Eqs. (8), with $Q=1$, and (9). Because of the poor resolution of such an inversion, required by this method to tame the ill-posed character of inversion calculations involving continuous distributions, their results did not yield an isolated discrete line for the $\phi_{e}(t)$ part of the response but instead led to an appreciably broadened continuous DRT in place of it. The LEVM program includes an inversion algorithm with much higher resolution, one that allows one to obtain single lines for discrete distributions and to distinguish continuous and discrete parts of a complete distribution. $^{40,41,46}$

Note that when $\tau_{s}$ exhibits Arrhenius behavior, the usual CSD1 situation, the cutoff quantity $y_{c}$ becomes

$$
y_{c}=-(k T)^{-1}\left[E_{s}+k T \ln \left(\tau_{s \infty} / \tau_{c}\right)\right] \text {, }
$$

somewhat reminiscent of Eq. (10). Since $\tau_{s \infty}$ will usually be appreciably less than $\tau_{c}$, it is possible for $y_{c}$ to become positive at a sufficiently high temperature.

To obtain an expression with which to calculate $\tau_{s}(T)$, Ngai and Rizos combined Eqs. (12), (13), and (16), using the guessed values $\beta_{0}=0.4$ and $\epsilon_{\infty}=15$. They gave the result

$$
\tau_{s}(T)=10^{-14.6} \exp (0.33 \mathrm{eV} / k T) \mathrm{s},
$$

where the sign of the exponent of their pre-exponential factor has been corrected. In contrast, direct calculation using $\beta$ $=\beta_{1}=0.6$ yields $\tau_{s}(T) \simeq 10^{-14.78} \exp (0.33 \mathrm{eV} / k T) \mathrm{s}$. But, as we shall see, neither result is adequate.

Consider now a much more appropriate calculation of $\tau_{s}(T)$ using the cutoff model. Although $\beta_{1}$ is often found to be temperature dependent, in the absence of further information we shall take it independent of temperature, as did NR for $\beta_{0}$. Further, we take its value as 0.5 , consonant with the KWW0 DRT expression of Eq. (11).

Kincs and Martin ${ }^{1}$ listed two $z=0 \quad \sigma_{\mathrm{dc}}$ values at $298 \mathrm{~K}$ : a value extrapolated from low-temperature results, $\sigma_{\text {dce }}$ $=0.0014(\Omega \mathrm{cm})^{-1}$, and the smaller actual value at that temperature, $\sigma_{\mathrm{dc} a}=0.0010(\Omega \mathrm{cm})^{-1}$. Only $\sigma_{\mathrm{dce}}$ was used by NR, but the use of both quantities allows one to avoid the need to make any arbitrary assumption about the value of the $\epsilon_{C \infty}$ quantity of Eq. (15). From Eqs. (15), (16) with $\beta=0.5$, and (17), it follows from the cutoff model that

$$
\sigma_{\mathrm{dce}} / \sigma_{\mathrm{dc} a}=2 \Gamma\left(\frac{3}{2}, x_{c} / 4\right) / \Gamma\left(\frac{1}{2}, x_{c} / 4\right) .
$$

Since the above ratio is 1.4 at $T=298 \mathrm{~K}$ for the $z=0$ material, we need only solve Eq. (20) for $x_{c}$ and so obtain $\tau_{s}(298$ $\mathrm{K})$ using $\tau_{c}=10^{-12} \mathrm{~s}$. A root-finding procedure yielded $\tau_{s}$ $=3.4724 \times 10^{-12} \mathrm{~s}$. This value and $E_{s}=0.33 \mathrm{eV}$ then led to $\tau_{s \infty} \simeq 9.114 \times 10^{-18} \mathrm{~s}$, much smaller than that in Eq. (19).

We may now solve Eq. (18) for the temperature at which $y_{c}=0$ and $\tau_{s}=\tau_{c}$. The result is about $330 \mathrm{~K}$. Finally, since all quantities in Eq. (15) are now known at $T=298 \mathrm{~K}$ out $\epsilon_{C \infty}$, it may be evaluated. The result found is 0.1098 , very much smaller than the NR value of $\epsilon_{\infty}=15$. In the absence of

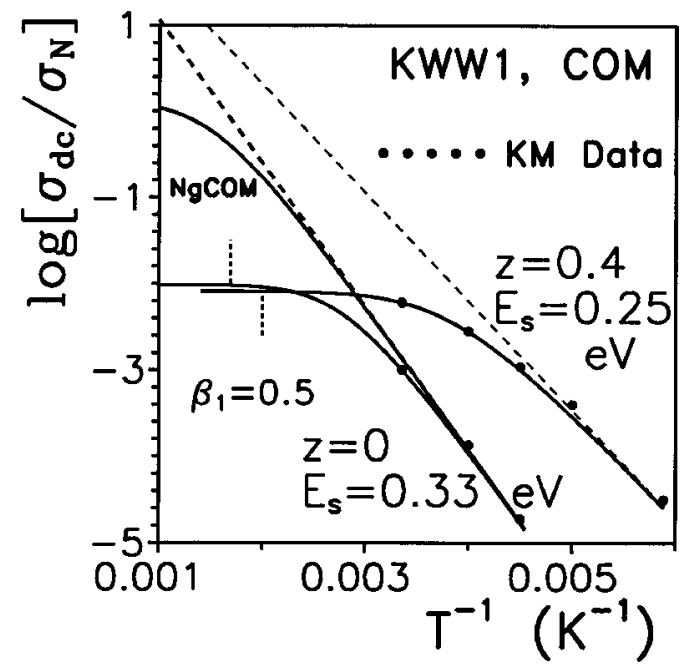

FIG. 2. Log plots of calculated $\sigma_{\mathrm{dc}}(T)$ curves versus $1 / T$ for $z \mathrm{AgI}$ $+(1-z) x\left(0.525 \mathrm{Ag}_{2} \mathrm{~S}+0.475 \mathrm{~B}_{2} \mathrm{~S}_{3}: \mathrm{SiS}_{2}\right)$ glass with $z=0$ and 0.4 . All results were calculated by the present $\mathrm{COM}$ approach with $\beta_{1}=0.5$. Glass transition temperatures are indicated by the vertical dashed lines. The $\mathrm{Ng}$ COM curve involves an $\epsilon_{C \infty}$ value of 15 , rather than its far smaller proper value, in order to illustrate some of the effects of the Ngai and Rizos choice of $\epsilon_{\infty}=15$. Here and hereafter, $\sigma_{N}=1(\Omega \mathrm{cm})^{-1}$.

additional information, which would be available from fitting the full data, we shall take $\epsilon_{C \infty}$ temperature independent, just as NR did for $\epsilon_{\infty}$. Had the original modulus-formalism expression of Eq. (13) used by NR been appropriate rather than Eq. (15), an estimate of $\epsilon_{\infty}$ smaller than unity would have been found by the above procedure, but it would have had to be rejected on physical grounds. On the other hand, since $\epsilon_{C \infty}$ is essentially just a conductive-system proportionality constant, as shown by Eq. (15), it may be either much smaller or even much larger than unity.

Now that an appropriate expression for $\tau_{s}(T)$ is available over the full temperature range, we can use the combination of Eqs. (15) and (17) to calculate $\sigma_{\mathrm{dc}}(T)=1 / \rho_{s}(T)$ and $\tau_{s}(T)$ "data" as functions of temperature. This has been done at $10^{\circ}$ intervals over the range $90 \leqslant T \leqslant 610 \mathrm{~K}$, and the above procedure has also been used to obtain results for $\mathrm{z}=0.4$ as well. Conductivity curves are presented in Fig. 2 for both the low-temperature-extrapolated Arrhenius behavior and the corresponding calculated non-Arrhenius response. Because of the density of calculated points, here and subsequently, only lines connecting the points are included, without the points themselves. The actual data points shown were obtained by scaling from an enlarged copy of the KM Fig. 2. Note that the data values shown at $T^{-1}=(298 \mathrm{~K})^{-1}$ were used in the calculations and so agree exactly with the corresponding calculated values. The small deviations apparent at the lower temperatures may arise from multiple causes: for example, the present assumption of temperatureindependent $\beta_{1}$ and $\epsilon_{C \infty}$, errors in the graphical estimation by $\mathrm{KM}$ of the dc conductivities at each temperature, errors in the present scaling procedure, and possible small differences between the actual activation energies for $z=0$ and 0.4 and those estimated by KM and used herein.

Incidentally, for $z=0.4$ where $E_{s}=0.25 \mathrm{eV}, \epsilon_{C \infty}$ was found to be about 0.0919 , surprisingly close to the above 


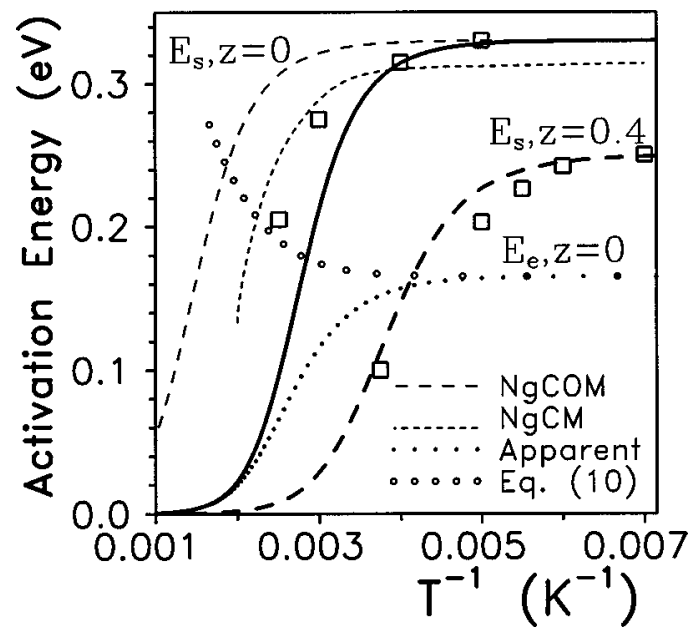

FIG. 3. Temperature variation of the apparent $E_{s}$ activation energy for $z$ $=0$ and 0.4 , solid and long-dashed lines, respectively. Also, temperature variation of the $z=0$ apparent $E_{e}$ Debye-response activation energy, and its actual behavior calculated using Eq. (10). The NgCOM curve is derived from that of Fig. 2, and the $\mathrm{NgCM}$ curve is a smoothed version of that presented by Ngai and Rizos (Ref. 2). The large open squares show values estimated by Kincs and Martin from their data. Here $\beta_{1}=0.5$ for the COM curves and $\beta_{0}=0.4$ for the $\mathrm{NgCM}$ results.

$z=0$ value. Figure 2 also includes a curve marked $\mathrm{NgCOM}$, the outcome of a $z=0$ calculation of the present cutoffmodel type using a constant value of $\epsilon_{C \infty}=15$, in order to illustrate the deleterious effect of such a large value. For completeness, all the curves have been extended beyond the $T=T_{g}$ points, ones designated by the vertical dotted lines.

Numerical differentiation has been used to calculate the apparent activation energy, $E_{\text {sap }}$, curves from the present $\sigma_{\mathrm{dc}}(T)$ calculated curves, and the results are illustrated by the solid and long-dashed lines in Fig. 3. In addition, Fig. 3 includes curves derived from the $\mathrm{NgCOM}$ results of Fig. 2, and a smoothed curve, designated $\mathrm{NgCM}$, the result of the NR coupling model calculation. ${ }^{2}$ It is not clear why the $\mathrm{NgCM}$ curve approaches $0.31 \mathrm{eV}$ rather than the proper value of $0.33 \mathrm{eV}$ at low temperatures. Further, this curve reaches an implausible high-temperature limiting value of $E_{s} \beta_{0}=0.33 \times 0.4=0.132 \mathrm{eV}$, rather than continuing to decrease smoothly toward zero as the temperature increases.

Also shown in Fig. 3 are a few apparent activation energy points (large open squares) scaled from Fig. 3 of Kincs and Martin. ${ }^{1}$ Although the present solid-line and long-dashed COM curves and these $\mathrm{KM}$ points are in semiquantitative agreement, there are several reasons, in addition to those listed above in connection with the Fig. 2 results, for the remaining discrepancies: (a) the present work is a simulation, not a fit of extensive data; (b) differentiation magnifies errors; the 0.5 value of $\beta_{1}$ used here is unlikely to be fully appropriate, especially since $\beta_{1}$ may be temperature dependent; and (c), in obtaining their values of $\sigma_{\mathrm{dc}}$, KM apparently took no account of possible electrode effects. ${ }^{42,45}$ Most of these same limitations, or their CM equivalents, apply to the NR analysis as well. Thus, although it seems an exaggeration to claim, as NR have done, that their application of the $\mathrm{CM}$ to the present $z=0$ data reproduces the nonArrhenius temperature dependence of the KM data in its en- tirety, their work on the problem was nevertheless seminal.

Figure 3 also includes a dotted curve of $E_{\text {eap }}$, the common apparent activation energy of the Debye-region quantities $\rho_{e}$ and $\tau_{e}$, which are connected by Eq. (15) with $\langle x\rangle_{0}$ $=1$ and the " $s$ ", subscripts changed to " $e$." See the next section for a discussion of the calculation of these quantities with the CM or COM. As expected, the low-temperature asymptotic limit of the $E_{\text {eap }}$ curve for the $z=0$ case is 0.33 $\times 0.5=0.165 \mathrm{eV}=E_{e 0}$. Finally, Fig. 3 also includes a curve of the $E_{e}(T)$ activation energy, calculated using Eq. (10). Unlike the apparent $E_{s}$ curve, we see that it increases with temperature rather than remaining temperature independent. This is, of course, an indication of the unsuitability of the Arrhenius equation at high temperatures and is intimately linked with the approach to saturation of $\sigma_{\mathrm{dc}}(T)$ at high temperatures.

It is important to emphasize the distinction between apparent and actual activation energies for the present situation. If there were no cutoff effects present, it would be necessary for $E_{s}$ to increase, not decrease, with increasing temperature, in order to explain the approach to saturation of the conductivity. For the $z=0$ situation, $E_{s}$ would then have to be about $0.40 \mathrm{eV}$ at $400 \mathrm{~K}$ and $0.56 \mathrm{eV}$ at $590 \mathrm{~K}$ to explain the observed saturation. Kincs and Martin have suggested that the saturation effects in their data might be associated with a temperature-dependent ionic mobility in the region where all available mobile ions are fully dissociated. But such a full dissociation, in the absence of cutoff, would lead to a limiting conductivity of more than $530(\Omega \mathrm{cm})^{-1}$, as shown by the high-temperature limit of Eq. (12).

While some temperature-dependent mobility effects cannot be ruled out by the present analysis, its good prediction of the approach to saturation, based entirely on cutoff at $\tau_{c}$, an effect unconnected with temperature-dependent mobility or $E_{s}$ activation energy changes, strongly suggests that cutoff is likely to be the cause of the observed approach to saturation. If so, it appears that the only practical way to obtain higher high-temperature conductivity is to find or create a material with a smaller value of $\tau_{c}$, clearly not an easy task.

A comparison of the saturated conductivities shown in Fig. 2 for the $z=0$ and 0.4 values indicates that the lower value of $E_{s}$ of the latter material does not lead to higher limiting conductivity, although if $\epsilon_{C \infty}$ were larger, a higher saturation value would be achieved, as shown by the $\mathrm{Ng}$ COM curve of Fig. 2. But $\epsilon_{C_{\infty}}$ is not a separately disposable quantity, as confirmed by Eq. (15), which demonstrates that at low temperatures $\epsilon_{C \infty}$ is determined by the $\rho_{s} / \tau_{s}$ ratio for a given value of $\beta$. Although it might appear from Eq. (15) that to obtain maximum dc conductivity at a given temperature $\tau_{s}$ should be as small as possible, decreasing $\tau_{s}$ increases $y_{c}$ and thus $\langle x\rangle_{0}$, resulting in a compensating effect. It thus seems that higher conductivity requires either a smaller temperature-independent value of $\tau_{c}$ or a $\tau_{c}$ that decreases with increasing temperature, neither choice a readily controllable possibility. Even though their application of the $\mathrm{NgCM}$ to explain the non-Arrhenius effects observed by KM is imperfect, Ngai and Rizos deserve great credit for first suggesting that the effect in fast ionic conductors is associated with the influence of a nonzero $\tau_{c}$. 
TABLE I. Calculated and fitting frequency-domain results for $z=0, T$ $=200 \mathrm{~K}$, KWW situations with $\beta=0.5$ and $\tau_{c}=10^{-12} \mathrm{~s}$. Here and elsewhere, $\rho_{N}=1 \Omega \mathrm{cm}$ and $\tau_{N}=1 \mathrm{~s} . n=1:$ KWW1; $n=0$ : KWW0.

\begin{tabular}{ccccc}
\hline \hline $\mathrm{A}$ & $\mathrm{B}$ & $\mathrm{C}$ & $\mathrm{D}$ & $\mathrm{E}$ \\
& Type, $n$ & $\mathrm{COM}$ & $\mathrm{CM}$ & COM fit of CM \\
\hline$\rho_{s} / \rho_{N}$ & 0,1 & $3.928 \times 10^{5}$ & $3.928 \times 10^{5}$ & $3.925 \times 10^{5}$ \\
$\tau_{s} / \tau_{N}$ & 0,1 & $1.885 \times 10^{-9}$ & $1.885 \times 10^{-9}$ & $1.875 \times 10^{-9}$ \\
$y_{c}$ & 1 & -7.542 & -60 & -7.756 \\
& 0 & -7.542 & -60 & -8.708 \\
$\left\langle x^{-1}\right\rangle_{1}$ & 1 & 0.4935 & 0.4935 & 0.4942 \\
$\langle x\rangle_{0}$ & 0 & 2.026 & 2.000 & 2.015 \\
$\rho_{e} / \rho_{N}$ & 1 & $\left(7.974 \times 10^{3}\right)$ & $7.144 \times 10^{3}$ & $\ldots$ \\
& 0 & $\left(1.490 \times 10^{4}\right)$ & $1.114 \times 10^{4}$ & $\ldots$ \\
$\tau_{e} / \tau_{N}$ & 1 & $\left(7.752 \times 10^{-11}\right)$ & $6.955 \times 10^{-11}$ & $\ldots$ \\
& 0 & $\left(7.940 \times 10^{-12}\right)$ & $9.819 \times 10^{-13}$ & $\ldots$ \\
$Q$ & 1 & 1.786 & 1.602 & $\ldots$ \\
& 0 & 0.0677 & 0.0226 & $\ldots$ \\
$\gamma_{x}$ & 1 & 0.2268 & 0.4438 & $\ldots$ \\
& 0 & 6.771 & 8.965 & $\cdots$ \\
\hline \hline
\end{tabular}

\section{COMPARISON OF CUTOFF AND COUPLING MODEL PREDICTIONS}

\section{A. General}

Most of the comparisons in this section deal with frequency rather than with the temporal response, and they all involve the results of the $z=0$ calculations of the last section. Until now, the CM has only been defined at the temporal level; thus, it is appropriate to consider how it might be defined in the frequency domain. Although COM time dependence has already been illustrated in Fig. 1 for KWW0 and KWW1 models with and without cutoff, some comparison in the time domain between COM and CM dependencies is worthwhile and will be presented first. Because of the high frequencies or short times involved in the Debye part of the $\mathrm{CM}$ or COM response, there has, so far, been little or no detailed fitting of data with either of these models in the range near $\omega_{c}$ or $\tau_{c}$. This is one reason why the results in the preceding section are important: they explain an interesting and relatively common observed effect using the COM approach without the need for data actually extending up to $\omega_{c}$ or down to $\tau_{c}$.

The range of expressions for and values of $Q$ that have appeared implicitly or explicitly in previous $\mathrm{NgCM}$ work might be thought to make its actual application ambiguous in situations where $\tau_{e}$ is not determined directly from data fitting, i.e., in most previous $\mathrm{NgCM}$ studies. For example, Eq. (6) shows that without knowledge of $Q, \tau_{e}$ cannot be determined, or vice versa. NR have avoided this problem in the work of Ref. 2 by using the modern ${ }^{52}$ version of the $\mathrm{NgCM}$, one where in Eq. (6), $Q=1$ by hypothesis. ${ }^{36}$ It should be recalled that this choice of $Q$ only applies for the KWW0 (or KWWD) response, not for the KWW1 time or frequency response; see Sec. III C and Table I for details.

Ngai and Rizos have presented a form of Eq. (13) for $\tau_{e} \leqslant \tau_{c}$, with the equation rewritten for $\tau_{e}$ rather than for $\tau_{s}$ and with $\langle x\rangle_{0}=1$. Although these choices are appropriate for the exponential response region, there remain several problems with this approach: the use of $\epsilon_{\infty}$ instead of $\epsilon_{C \infty}$; the implicit identification of $\rho_{e}$ with $\rho_{s} \equiv \sigma_{\mathrm{dc}}^{-1}$; and the condition $\tau_{e} \leqslant \tau_{c}$ itself. In the calculation of $\tau_{e}(T)$ for the $z=0$ situation of the previous section, it was found that $\tau_{e}$ approaches $\tau_{c}$ asymptotically as the temperature increases, and it is $35 \%$ larger than $\tau_{c}$ at $T=400 \mathrm{~K}$ and is still $2 \%$ larger at $500 \mathrm{~K}$. This behavior is physically plausible. If $\tau_{c}$ is the primitive $(\mathrm{NgCM})$ or limiting $(\mathrm{COM})$ response time of the system, there should be no smaller response time for the overall relaxation process considered.

The above behavior of $\tau_{e}(T)$, which applies for either the temporal or frequency-domain response, as discussed below, means that $\tau_{e}(T)$, like $\rho_{s}(T)$, can only exhibit an Arrhenius response with a temperature-independent $\tau_{e \infty}$ and $E_{e}$ at low temperatures. By contrast, the conventional $\mathrm{NgCM}$ treatment involves Arrhenius behavior of $\tau_{e}(T)$ over the full temperature range. The derivation of Eq. (10) assumes that the deviation from Arrhenius behavior involves changes in $E_{e}$, but, alternatively, one could take $E_{e}$ temperature independent and the $\tau_{e \infty}$ variable if one insisted on using the Arrhenius equation at all at high temperatures. Incidentally, although $\rho_{e}$ indeed approaches $\rho_{s}$ asymptotically, it is always smaller than $\rho_{s}$, about $8 \%$ smaller at $T=400 \mathrm{~K}$ for the $z=0$ calculations. More details are provided later.

\section{B. Time-domain response}

In the temporal domain, there are several ways to obtain needed $Q$ or $\tau_{e}$ values at each temperature of interest when the $Q=1$ choice is not appropriate. First, one could fit the KWW0 or KWW1 COM response directly to actual temporal or frequency response data. If the data allowed $\tau_{c}$ to be adequately estimated or if it were known independently, such an analysis would usually be sufficient. Although knowledge of $\tau_{e}$ is not needed for COM fitting, it could be obtained by separate fitting of the short-time or high-frequency response region, as already discussed. With such a value available, a corresponding CM response could be calculated using Eqs. (8) and (9) for the KWW0 temporal-response situation. To obtain the KWW1 CM response, one would use Eq. (9) and match its value at $t=\tau_{c}$ with the calculated KWW1 response without a cutoff. In the present work, the exact $\mathrm{KWW} n$ COM time response is calculated using the appropriate $G_{n}$ DRT.

Some time domain, $\beta_{0}=\frac{1}{3}$, COM and CM results at $T=340 \mathrm{~K}$ for KWW0 and KWW1 are compared in Fig. 4. Here, as usual, $\tau_{c}=10^{-12} \mathrm{~s}$. Figure $4(\mathrm{a})$ is plotted so that the exponential response yields a straight line, and its range is limited to the region $0 \leqslant t / \tau_{c} \leqslant 2$ in order to show the CM transition clearly. For both KWW0 and KWW1, $y_{c} \simeq 0.343$; and $\tau_{e}$ and $Q$ are about $1.051 \times 10^{-11} \mathrm{~s}$ and 12.48 for KWW0, and $3.186 \times 10^{-12} \mathrm{~s}$ and 3.186 for KWW1, respectively. For comparison, the KWW1 $Q$ value in the frequency domain is about 2.531 at this temperature. The present results, besides illustrating the large differences in $\tau_{e}$ between KWW0 and KWW1 situations, clearly show the much greater CM discontinuity in slope at $t=\tau_{c}$ for KWW0 as compared to KWW1; thus, KWW1 analysis characteristically leads to much closer agreement between the COM and CM approaches than does KWW0. 

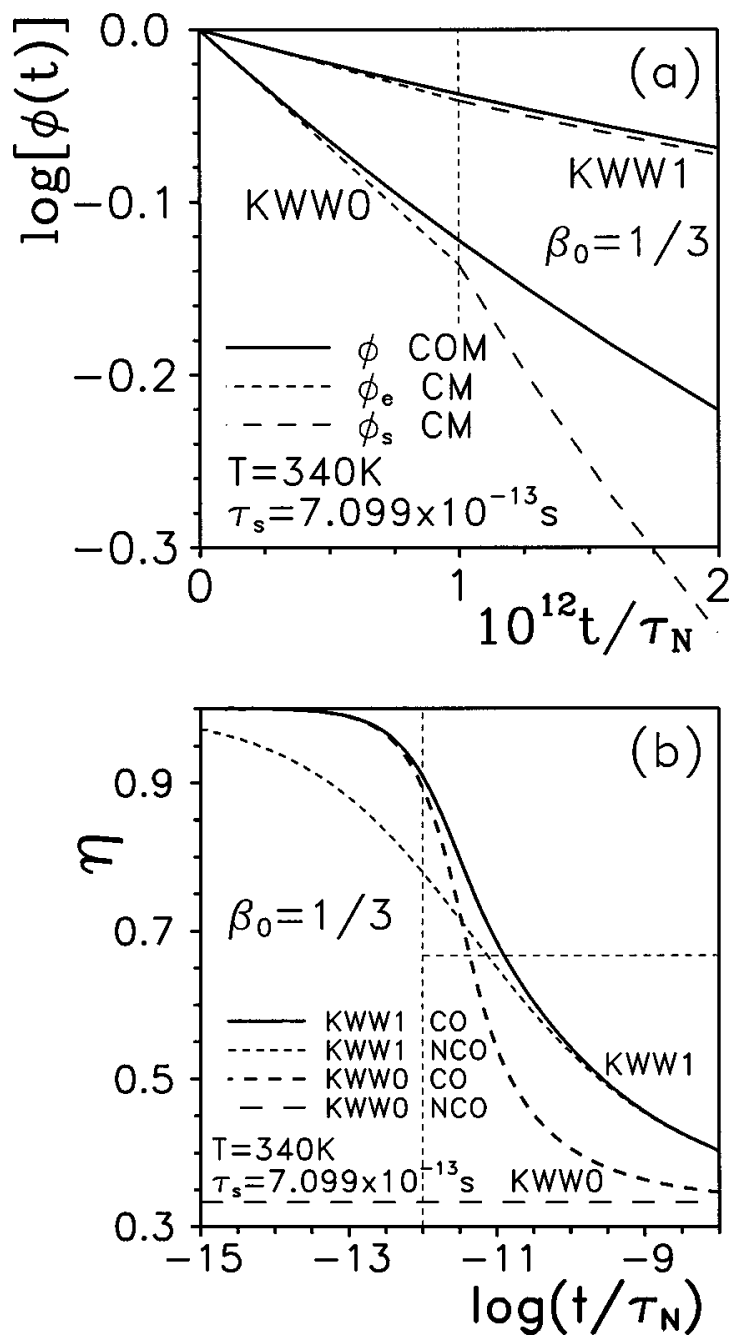

FIG. 4. (a) A comparison of COM and CM temporal response curves for KWW0 and KWW1 situations, showing response near the CM transition at $t=\tau_{c}=10^{-12} \mathrm{~s}$. (b) A longer-time comparison of KWW0 and KWW1 slope response with $(\mathrm{CO})$ and without $(\mathrm{NCO})$ cutoff. The curves marked $\mathrm{CO}$ involve a COM response. The approach to the KWW0/NCO stretchedexponential behavior (where $\eta=\beta_{0}$ ) is relatively slow for the other curves. Here and hereafter, $z=0$, and a vertical dashed line indicates the position of $\tau_{c}$ or $\omega_{c}$. The horizontal dashed line in this figure is plotted at $\eta=\frac{2}{3}$.

When the present KWW0 and KWW1 COM results are plotted versus $\left(t / \tau_{N}\right)^{1 / 3}$; curves similar in shape to the corresponding ones of Fig. 1 are obtained. But since they do not yield precise information on how closely $\phi(t)$ conforms with stretched-exponential behavior, some of the results of a different analysis are presented in Fig. 4(b). Let us define

$$
\eta \equiv d \log \{-\ln [\phi(t)]\} / d \log \left(t / \tau_{N}\right) .
$$

If $\phi(t)$ is given by the stretched exponential of Eq. (5), then $\eta=\beta$, a result that is indeed obtained over the full time range for the KWW0 response without cutoff.

Figure 4(b) shows $\eta$ behavior for the usual four choices. First, note that $\eta$ is not independent of time for the top three responses shown in the figure, demonstrating that the stretched-exponential form is inapplicable for these three response possibilities. Second, note that if we set $\eta=\beta_{1}$ for the KWW1 situations, $\beta_{1}$ is not close to the value $\frac{2}{3}$, which might be expected if $\eta$ were a proper KWW or power-law exponent. ${ }^{45,46} \mathrm{Next}$, one sees that the presence or absence of cutoff makes little difference in the KWW1 response for $t / \tau_{c} \gg 1$. Incidentally, a nonlinear-least-squares, stretchedexponential fit of the KWW1 COM data over the range $10^{-13} \leqslant t / \tau_{N} \leqslant 10^{-11}$ yielded quite a good fit, with estimates of $\tau$ and $\beta$ of about $2.1 \times 10^{-11}$ s and 0.79 , respectively, with one to two percent estimated uncertainties, showing that the results of such a fitting may be strongly misleading.

Although both the KWW0 response with cutoff and the KWW1 response approach $\beta_{0}$ asymptotically, the former does it appreciably faster. For the present temperature, the cutoff KWW0 situation yields $\phi(t)$ values of about 3 $\times 10^{-5}$ and $8 \times 10^{-11}$ at $t=10^{-9}$ and $10^{-8} \mathrm{~s}$, respectively, while the KWW1 values are about $10^{-3}$ and $10^{-8}$, respectively. It is thus clear that ordinary temporal measurements, which usually do not extend to very small values of $\phi(t)$, will not allow one to reach values of $\eta$ close to $\beta_{0}$ for either type of response. But only when $\eta=\beta_{0}$ does one have true stretched-exponential behavior.

\section{Frequency-domain response}

Coupling may be achieved in the frequency domain by setting a frequency response function, evaluated at $\omega_{c}$, equal to the Debye response at this frequency. Such coupling has not been considered previously for the CM. Both real and imaginary parts must be matched at the transition point. If we make the usual ${ }^{42,45-47}$ assumption that $\rho_{n}(\infty)=0$, and consider only the KWW response, an unnecessary limitation, but one consistent with the time-domain $\mathrm{NgCM}$, then with $\rho_{n}(0) \equiv \rho_{s n}$, frequency-response coupling is achieved if

$$
\rho_{n}\left(\omega_{c}\right)=\rho_{s n} I_{n}\left(\omega_{c}\right)=\rho_{e} /\left(1+i \omega_{c} \tau_{e}\right),
$$

a relation sufficient to allow values of both $\rho_{e}$ and $\tau_{e}$ to be obtained when the complex quantity $I_{n}\left(\omega_{c}\right)$ is known. Although rapid and accurate calculation of the KKW $n$ response in the frequency domain has not been possible in the past for arbitrary $\beta_{n}$, a procedure to do so has recently been added to the latest version of the LEVM complex nonlinear-leastsquares fitting program, ${ }^{40}$ as well as one to implement Eq. (22) for the noncutoff $\mathrm{KWW}$ response at $\omega \leqslant \omega_{c}$. Thus, given values of $\rho_{s n}, \tau_{s n}, \tau_{c}$, and $\beta_{n}$, one can readily calculate the corresponding $\rho_{e}, \tau_{e}, Q$ [from Eq. (6)], and $\gamma_{x}$ values and then, if desired, use the results to model the full response. For the present $z=0$ situation, one finds that $\tau_{e \infty}$ $=5.35093 \times 10^{-15} \mathrm{~s}$, and for sufficiently small temperatures,

$$
\begin{aligned}
\rho_{e}(T) & =\rho_{e \infty} \exp \left(E_{e 0} / k T\right) \\
& =0.550358 \exp (0.165 \mathrm{eV} / \mathrm{kT})(\Omega \mathrm{cm}) .
\end{aligned}
$$

The CM matching defined by Eq. (22) leads to a discontinuity in slope at $\omega=\omega_{c}$, but matters differ between CSD0 (or DSD) and CSD1 situations. With matching between the dispersed CSD0 response and the Debye response, both the real and imaginary parts of the complex resistivity response show slope discontinuities when no separate account is taken of $\epsilon_{D \infty}$, as in Eq. (22). On the other hand, for the more reasonable CSD1 situation, it turns out that only the real part 
shows such a discontinuity, whether or not the effect of $\epsilon_{D \infty}$ is included in the response at the match point. This fortunate result follows because the high-frequency CSD1 imaginary response without cutoff already involves ${ }^{45,47}$ the proper physically required limiting slope of -1 . In order to implement Eq. (22)-matching properly for actual data, one should first fit the data in the region $\omega \ll \omega_{c}$, taking proper account of $\epsilon_{D \infty}$. This would then allow the quantities $\rho_{s n}$ and $I_{n}(\omega)$ to be obtained, and Eq. (22) could then be applied if $\omega_{c}$ were known independently or if it could be determined from the data. Note that although $\tau_{s}$ can be smaller than $\tau_{c}$ at frequencies above $\omega_{c}$, the CM or COM response in this region is dominated by Debye behavior.

The above procedure is unnecessary for the COM. For this approach, the amount of cutoff is determined by the $\tau_{s} / \tau_{c}$ ratio, as discussed in the last section, with no cutoff effects apparent when $\tau_{s} / \tau_{c} \gg 1000$ and with a transition to the situation where the full response approaches single-timeconstant Debye behavior for $\tau_{s} / \tau_{c} \ll 1$. In fitting actual data, one would use an appropriate expression for $I_{n}(\omega)$ associated with a known DRT and calculated employing Eq. (1). By allowing the DRT cutoff parameter to be one of the free parameters of the fit, one could then obtain an estimate of the appropriate $\tau_{c}$ value, provided the experimental frequency range was adequate.

As an example, consider the $z=0, T=200 \mathrm{~K}$ frequencyresponse situation with the usual choice of $\tau_{c}=10^{-12} \mathrm{~s}$. The KWW1 and KWW0 cutoff-model parameter values for these choices are presented in column C of Table I. The $y_{c}$ value of about -7.5 leads here to a limiting Debye response for $\beta>\omega_{c}$, without the need for grafting on any separate Debye response as in the CM. Since the COM transition to exact Debye behavior is gradual rather than abrupt, the cutoff data were fitted, using LEVM, to the Debye model over the frequency region from $10^{14}$ to $10^{16} \mathrm{~Hz}$, yielding a virtually perfect fit that led to the parenthesized $\rho_{e}$ and $\tau_{e}$ values shown in column C. Incidentally, these quantities led, through the analog of Eq. (15) for a Debye response, to the same $\epsilon_{C \infty}$ value as that involved in the low-frequency response, showing, as expected, that the response beyond the cutoff frequency is still an integral part of the total KWW1 behavior.

Coupling-model results are shown in column D of Table I. The large $y_{c}=-60$ value ensures that calculated KWWn response data up to $\omega=10^{12} \mathrm{~Hz}$ involves no cutoff effects, and the subsequent matched Debye response leads, through Eq. (22), to the $\rho_{e}$ and $\tau_{e}$ values shown, ones reasonably close to those for the COM, except for the KWW0 $\tau_{e}$ value. In contrast, the column $\mathrm{E}$ values are those obtained from a LEVM fitting of the COM to the CM data associated with column D. We see that all the parameter values agree closely with those of column $\mathrm{C}$, except $y_{c}$. This substantial agreement indicates that there are only minor differences between the COM and CM responses, except near the coupling transition point. Note that although the bottom lines of columns C and D show KWW1 $Q$ and $\gamma_{x}$ values that are not greatly different from $Q_{\mathrm{Ng}}$ and $\gamma$, the KWW0 ones are very different, indicating that CM slope discontinuities will be appreciably smaller for the KWW1 than for the KWW0 situations.

Figure 5 shows the $\log -\log$ frequency response of the



FIG. 5. The coupling-model frequency response for the real and imaginary parts of the complex resistivity, $\rho=\rho^{\prime}-i \rho^{\prime \prime}$. Here and elsewhere $\rho_{N}$ $=1 \Omega \mathrm{cm}$.

KWW1 complex conductivity defined by the CM parameters in column D of Table I. Notice that although there is an abrupt change in the slope of $\rho^{\prime}(\omega)$ from ${ }^{45-47}-\left(1+\beta_{1}\right)$ $=-1.5$ to the limiting Debye slope of -2 , there is no transition in the $-\rho^{\prime \prime}(\omega)$ slope of -1 . Thus, we see that the presence or absence of coupling to the final Debye response makes a negligible difference in a KWW1 $-\rho^{\prime \prime}(\omega)$ curve for frequencies appreciably beyond its peak response.

Because the break in the $\rho^{\prime}$ slope, present at the CM transition point, does not show up strongly on a small $\log -$ $\log$ plot of $\rho^{\prime}$ vs $\omega$ and COM and CM results are hard to distinguish in such a plot, Fig. 6 presents the relative residuals, $r^{\prime} \equiv\left(\rho_{\mathrm{CM}}^{\prime}-\rho_{\mathrm{COM}}^{\prime}\right) / \rho_{\mathrm{CM}}^{\prime}$ and $r^{\prime \prime} \equiv\left(\rho_{\mathrm{CM}}^{\prime \prime}-\rho_{\mathrm{COM}}^{\prime \prime}\right) / \rho_{\mathrm{CM}}^{\prime \prime}$, for the $T=200 \mathrm{~K}$ fit and the corresponding $r^{\prime}$ one for $400 \mathrm{~K}$. Here, the CM subscript denotes the exact coupling-model data and the COM subscript indicates the complexnonlinear-least-squares fit predictions arising from the use of the KWW1 COM fitting model, as in column E of Table I. It

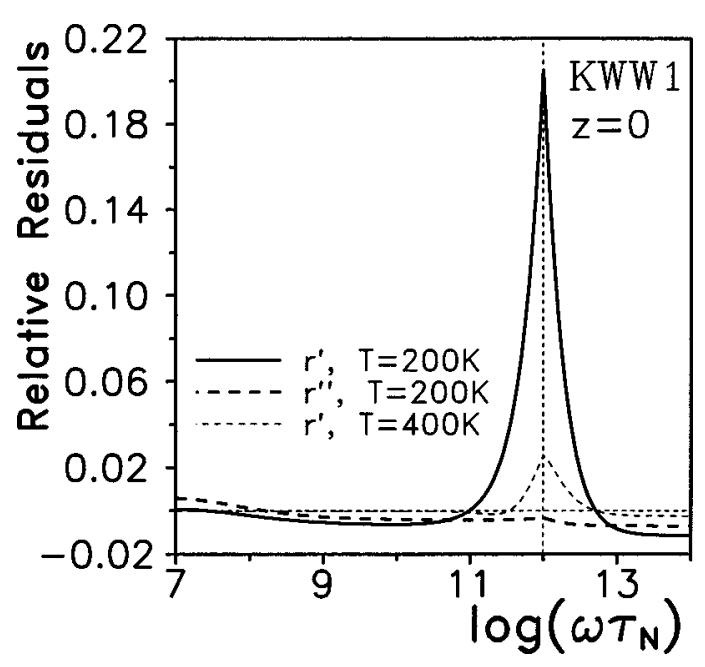

FIG. 6. Relative residuals for the real and imaginary parts of the complex resistivity resulting from the KWW1 COM fit (column E, Table I) of the $\mathrm{CM}$ response of column D of the table shown in Fig. 5. 




FIG. 7. Frequency response of the conductivity, $\sigma^{\prime}(\omega)=\operatorname{Re}[1 / \rho(\omega)]$, for the CM response of column D of Table I and the COM fit to it (column E of Table I).

is clear that while there can be a large relative error present in the real residuals, about $21 \%$ at the $\omega=\omega_{c}$ peak, there is practically negligible difference between the fit and the model for the imaginary-part residuals. Thus, the major difference here between the $\mathrm{CM}$ and COM frequency-response predictions is that the real part of the CM response can show a large abrupt change in slope in the neighborhood of $\tau_{c}$, while the corresponding COM curve shows only a gradual slope change between that appropriate for the KWW1 model without cutoff, $-(1+\beta)$ and the -2 value for Debye response. The maximum $r^{\prime}$ value at $T=400 \mathrm{~K}$ is much smaller because at this temperature, $y_{c} \simeq 2.03$, quite different from the value of -7.54 at $T=200 \mathrm{~K}$.

Another way of illustrating the effect of the slope discontinuity in the CM response is presented in Fig. 7. Here the COM (column E of Table I) and the CM (column D of Table I) $\sigma^{\prime}(\omega)$ responses are compared. Note that both curves show the approach to a final plateau at high frequencies.

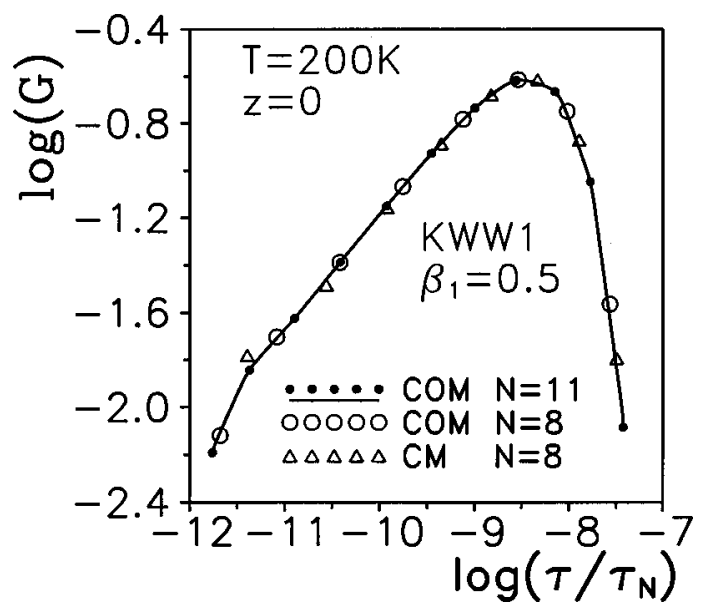

FIG. 8. Inversion results for the distributions of relaxations times associated with the $T=200 \mathrm{~K} \mathrm{KWW} 1$ frequency response defined in Table I for COM and $\mathrm{CM}$ situations.

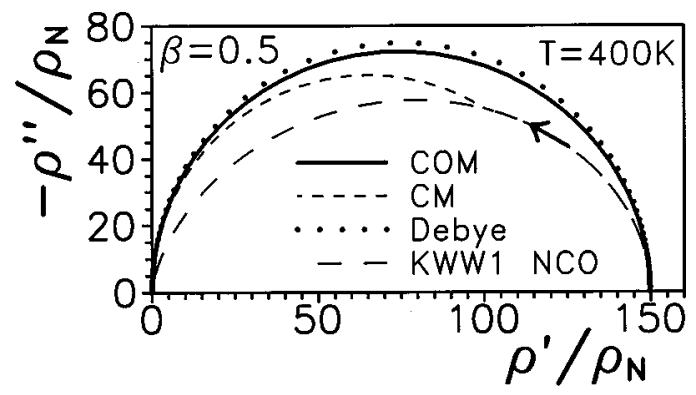

FIG. 9. Complex-plane plots of the complex resistivity for several different KWW1 situations. The arrow shows the direction of increasing frequency.

Figure 8 shows DRT estimates obtained by using LEVM to invert the frequency response data of columns $\mathrm{C}$ and $\mathrm{D}$ of Table I. First, we see that since the COM points appear in different positions on the curve for $N=8$ and for $N=11$ total inversion points, the distribution is continuous, not discrete. ${ }^{41,42,46}$ Second, the slope of the left, straight-line part of the curve is just $\beta_{1}=0.5$, as it should be. Third, as $N$ increases, the smallest- $\tau$ point approaches closer and closer to $\tau_{c}$ and exhibits smaller and smaller strength. But no matter how large $N$, no points appear for $\tau<\tau_{c}$, a requirement already mentioned.

Although the $G(x)$ distribution found from the COM response is clearly that for the KWW1 $G_{1}$ DRT with cutoff, one should not necessarily expect that the DRT found for the $\mathrm{CM}$ should be of the same form in the $\tau \approx \tau_{c}$ region. We see from the figure that in fact the results for the $\mathrm{CM}$ data begin to deviate from the $G_{1}$ curve as $\tau$ decreases toward $\tau_{c}$. Inversion is made difficult in this case by the discontinuity in slope at $\omega=\omega_{c}$, and no more than eight significant points could be obtained for it. Nevertheless, unlike the NR inversion results, ${ }^{2}$ no points or DRT density were found for $\tau$ $<\tau_{c}$. This is because the full CM dataset still involves a continuous distribution: the addition of a Debye response for $\omega \geqslant \omega_{c}$ with only a discontinuity in slope at the transition does not lead to a discrete response line at $\tau=\tau_{c}$, as it would if there were a region of no density between the low- and high-frequency (or short- and long-time) responses. This is further evidence that the Ngai identification of $\phi_{e}(\tau)$ as basic and fundamental may be inappropriate, at least in the $Q=1$ situation considered in Ref. 2 .

Another way of presenting frequency-response differences is illustrated in Fig. 9. Here complex-plane plots of the resistivity responses for various $T=400 \mathrm{~K} \mathrm{KWW} 1$ situations are shown. In this high-temperature case, $y_{c} \simeq 2.032$ for the COM curve, and the curve falls quite close to that of the single-relaxation-time Debye model. On the other hand, the $y_{c}=-60$ no-cutoff response is appreciably different. As expected, the low-frequency part of the CM curve agrees with the no-cutoff one, and the high-frequency part lies closer to the Debye curve, but the COM response is certainly more plausible than that of the CM here.

Figure 10 shows the dependence of $Q$ and $\left(\rho_{e} / \rho_{N}\right) /\left(\rho_{s} / \rho_{N}\right)^{0.5}$ on $T^{-1}$ and $y_{c}$ for the KWW1 $z=0$ situation. Here $Q$ varies from its low-temperature limiting value of about 1.773 to about 14 at $T=600 \mathrm{~K}$, quite different from the temperature-independent value of unity used by NR. ${ }^{2}$ The $\rho$ ratio, in the limit of low temperatures, is, from Eqs. 


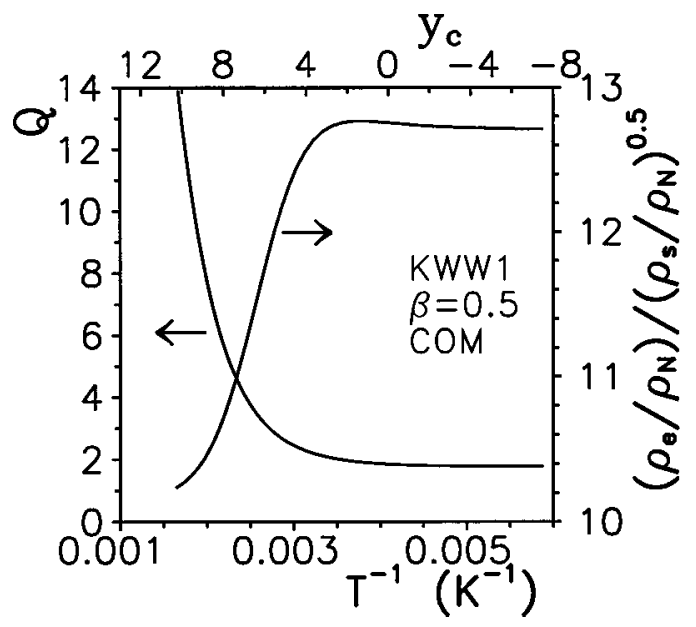

FIG. 10. Results showing the dependence of $Q$ and $\left(\rho_{e} / \rho_{N}\right) /\left(\rho_{s} / \rho_{N}\right)^{0.5}$ on $T^{-1}$ and on the cutoff parameter $y_{c}$.

(12) and (23), just $\left(\rho_{e \infty} / \rho_{N}\right) /\left(\rho_{s \infty} / \rho_{N}\right)^{0.5}$, equal to about 12.71 for the present situation. In the high-temperature limit, $\tau_{e}$ approaches $\tau_{c}$, and it has reached about $1.02 \times 10^{-12}$ by $T=600 \mathrm{~K}$. From the temperature-independent relation between $\rho_{e}$ and $\tau_{e}$, it follows that the high-temperature limit of $\rho_{e}$ is about $102.85 \Omega \mathrm{cm}$ for the present data. Finally, the frequency-response coupling of Eq. (22) leads to $\rho_{s}=\rho_{e}$ in the high-temperature limit since then only Debye behavior is present. At $600 \mathrm{~K}$ these quantities are 105.00 and 105.05 $\Omega \mathrm{cm}$, respectively, so the $\rho$ ratio is about 10.25 at this temperature and reaches a high-temperature limit of about 10.14.

Since at present no analytic expression for the DRT associated with the KWW response exists for arbitrary $\beta$, and closed-form DRT expressions are available ${ }^{63}$ for only a few specific values of $\beta$, the COM cannot be used for fitting data with a KWW model when $\beta$ is a free parameter of the fit, at least until V. 7.1 of the LEVM program is issued. This limitation does not apply to other response models whose DRTs are known, such as that of Davidson and Cole, but if fitting with a KWW approach is desired for data where it is important to take account of cutoff effects, the present results show that, especially for the KWW1, the CM defined here for the frequency domain could be used to provide an approximation to the full COM response.

\section{CONCLUSIONS AND IMPLICATIONS}

An important limitation in the time-domain Ngai coupling model has been identified and removed, and the model has been generalized to apply directly in the frequency domain without the need for Fourier transformation. The resulting CM frequency-response model and its COM counterpart require no knowledge of the important $Q$ parameter of Eq. (6) when data fitting allows a value of $\tau_{c}$ to be estimated, often possible with data limited to frequencies much less than $\tau_{c}^{-1}$. The Ngai coupling model involves a stretchedexponential KWW0 response in the time domain, but for thermally activated, conductive-system-dispersion situations, the more appropriate KWW1 response is not of a stretchedexponential form, except beyond the normal range of measurement. For both the frequency and temporal response of conductive systems, the KWW1 choice, which is now readily available in the LEVM fitting program, ${ }^{40}$ is much more appropriate than the KWW0 one for both the coupling model and the DRT cutoff model.

The coupling and cutoff relaxation approaches share the assumption that there exists a minimum relaxation or response time, $\tau_{c}$, but they differ in its provenance and interpretation. For the $\mathrm{NgCM}, \tau_{c}$ is a basic microscopic time that is associated with discrete simple exponential decay, taken as the fundamental relaxation process, and the dispersed response experimentally observed for $t \geqslant \tau_{c}$ or $\omega \leqslant \tau_{c}^{-1}$ is stated to be much less fundamental in this approach. Further, this latter response is required by the $\mathrm{NgCM}$ to be of either the stretched-exponential form in the time domain or of a type of associated KWW frequency response, here termed KWWD for dielectric systems or KWW0 for conductive ones.

In contrast, for the COM, $\tau_{c}$ is just the physically realizable minimum response time inherent in the primary relaxation process present. Although its presence also leads to a single-time-constant exponential or Debye response in the limiting short-time or high-frequency region, no separate discrete relaxation process needs to be assumed, as in the CM. When the full basic relaxation model is expressed in terms of a distribution of relaxation times, always mathematically possible, and, for most current models of interest, resulting in a continuous rather than a discrete distribution, $\tau_{c}$ is just the lower limit beyond which the distribution is zero. Further, cutoff can be applied for any response model, not just the stretched-exponential KWWO assumed in the $\mathrm{NgCM}$ analysis.

There seems to be good experimental evidence that $\tau_{c}$ plays an important role in relaxation, ${ }^{51,52}$ not surprising, since it is an always-present fundamental limiting response quantity. Not least of this evidence is the substantial agreement between the non-Arrhenius conductivity results of KM and the results of the present COM analysis, one that ascribes the departure from Arrhenius behavior observed at sufficiently high temperatures entirely to temperaturedependent cutoff effects. Although $\tau_{c}$ has generally been taken temperature independent, work needs to be done to show to what degree its value and temperature dependence may vary with different materials and thus with different relaxation processes, and, if possible, to derive expressions for it based on different possible specific microscopic relaxation processes. Such work would be desirable in order to substantiate or reject the plausible $\mathrm{NgCM}$ assumption that $\tau_{c}$ is associated with the dynamics of uncorrelated relaxing elements, an assumption likely to be consistent with the COM identification of $\tau_{c}$ as the minimum relaxation time present in the response. A beginning has been made for hopping conduction by the correlated jump model, ${ }^{59}$ a partly microscopic many-body approach but one that involves some empirical elements.

Although the present comparison of the predictions of the two models in the time and frequency domains might suggest that the $\mathrm{CM}$ is only an approximate form of the holistic COM, the philosophical underpinnings of the two models are sufficiently different that they should be consid- 
ered as separate theoretical approaches. Nevertheless, it seems plausible that most if not all of the good agreement of $\mathrm{NgCM}$ predictions with the experiment found by Ngai actually arises from intrinsic-cutoff $\mathrm{COM}$ behavior. Since the COM is both simpler in concept and more general than the $\mathrm{CM}$, and since it avoids the slope discontinuities of the latter, Occam's razor suggests that the COM approach should be preferred.

\section{ACKNOWLEDGMENTS}

I much appreciate the valuable suggestions of a referee and of Dr. K. L. Ngai.

${ }^{1}$ J. Kincs and S. W. Martin, Phys. Rev. Lett. 76, 70 (1996).

${ }^{2}$ K. L. Ngai and A. K. Rizos, Phys. Rev. Lett. 76, 1296 (1996).

${ }^{3}$ K. L. Ngai, A. K. Jonsher, and C. T. White, Nature (London) 277, 185 (1979).

${ }^{4}$ K. L. Ngai, in Fast Ion Transport in Solids, edited by P. Vashishta, J. N. Mundy, and G. K. Shenoy (Elsevier North-Holland, New York, 1979), p. 303.

${ }^{5}$ K. L. Ngai, Solid State Phys. 9, 127 (1979).

${ }^{6}$ K. L. Ngai and C. T. White, Phys. Rev. B 20, 2475 (1979).

${ }^{7}$ K. L. Ngai, Solid State Ionics 5, 27 (1981).

${ }^{8}$ S. Teitler, A. K. Rajagopal, and K. L. Ngai, Phys. Rev. A 26, 2906 (1982). Equation (5.3) for $g_{D}(\tau)$ is incorrect in this work. The power $\frac{1}{2}$ therein should be $-\frac{1}{2}$.

${ }^{9}$ K. L. Ngai and U. Strom, Phys. Rev. B 27, 6031 (1983).

${ }^{10}$ K. L. Ngai, R. W. Rendell, and H. Jain, Phys. Rev. B 30, 2133 (1984).

${ }^{11}$ K. L. Ngai, A. K. Rajagopal, and C. Y. Huang, J. Appl. Phys. 55, 1714 (1984).

${ }^{12}$ A. K. Rajagopal, S. Teitler, and K. L. Ngai, J. Phys. C 17, 6611 (1984).

${ }^{13}$ K. L. Ngai and A. F. Yee, in Relaxations in Complex Systems, edited by K. L. Ngai and G. B. Wright, Office of Naval Research (U.S. Government Printing Office, Washington, DC, 1985), p. 145.

${ }^{14}$ H. Jain and K. L. Ngai, in Ref. 13, p. 221.

${ }^{15}$ A. K. Rajagopal and K. L. Ngai, in Ref. 13 p. 275.

${ }^{16}$ R. W. Rendell and K. L. Ngai, in Ref. 13, p. 309.

${ }^{17}$ K. L. Ngai, in Non-Debye Relaxation in Condensed Matter, edited by T. V. Ramakrishnan and M. Raj Lakshmi (World Scientific, Singapore, 1985), p. 23.

${ }^{18}$ K. L. Ngai and A. K. Rajagopal, in Ref. 17, p. 387.

${ }^{19}$ K. L. Ngai and A. K. Rajagopal, Phys. Rev. Lett. 53, 1024 (1984).

${ }^{20}$ K. L. Ngai, A. K. Rajagopal, and S. Teitler, Physica A 133, 213 (1985).

${ }^{21}$ K. L. Ngai and H. Jain, Solid State Ionics 18\&19, 362 (1986).

${ }^{22}$ K. L. Ngai, R. W. Rendell, A. K. Rajagopal, and S. Teitler, IEEE Trans. Electr. Insul. EI-21, 313 (1986).

${ }^{23}$ K. L. Ngai, R. W. Rendell, A. K. Rajagopal, and S. Teitler, Ann. (N.Y.) Acad. Sci. 484, 150 (1986).

${ }^{24}$ K. L. Ngai, A. K. Rajagopal, and S. Teitler, J. Chem. Phys. 88, 5086 (1988).

${ }^{25}$ K. L. Ngai and R. W. Rendell, Phys. Rev. B 38, 9987 (1988).

${ }^{26}$ G. Balzer-Jollenbeck, O. Kanert, H. Jain, and K. L. Ngai, Phys. Rev. B 39, 6071 (1989).

${ }^{27}$ K. L. Ngai, J. N. Mundy, H. Jain, O. Kanert, and G. Balzer-Jollenbeck, Phys. Rev. B 39, 6169 (1989).

${ }^{28}$ K. L. Ngai and S. W. Martin, Phys. Rev. B 40, 10550 (1989).
${ }^{29}$ K. L. Ngai, J. Non-Cryst. Solids 131-133, 80 (1991).

${ }^{30}$ K. L. Ngai and O. Kanert, Solid State Ionics 53-56, 936 (1992).

${ }^{31}$ K. L. Ngai, S. L. Peng, and K. Y. Tsang, Physica A 191, 523 (1993).

${ }^{32}$ K. L. Ngai, J. Chem. Phys. 98, 6424 (1993). The second $<$ in Eq. (4) should be a $>$.

${ }^{33}$ K. L. Ngai, in Disorder Effects on Relaxational Processes, edited by R. Richert and A. Blumen (Springer-Verlag, Berlin, 1994), p. 29. The sign of the right side of Eq. (4.2) in this work should apparently be reversed.

${ }^{34}$ K. L. Ngai, C. M. Roland, and G. N. Greaves, J. Non-Cryst. Solids 182, 172 (1995).

${ }^{35}$ K. L. Ngai, J. Non-Cryst. Solids 203, 232 (1996).

${ }^{36}$ K. Y. Tsang and K. L. Ngai, Phys. Rev. E 54, R3067 (1996).

${ }^{37}$ K. Y. Tsang and K. L. Ngai, Phys. Rev. E 56, R17 (1997).

${ }^{38}$ C. M. Roland and K. L. Ngai, J. Non-Cryst. Solids 212, 74 (1997).

${ }^{39}$ R. V. Chamberlin, G. Mozurkewich, and R. Orbach, Phys. Rev. Lett. 53, 1510 (1984).

${ }^{40}$ J. R. Macdonald and L. D. Potter, Jr., Solid State Ionics 23, 61 (1987); J. R. Macdonald, J. Electroanal. Chem. 307, 1 (1991). A new version of the extensive LEVM fitting program, V. 7.01, may be obtained at no cost from Solartron Instruments, Victoria Road, Farnborough, Hampshire, GU147PW, England; electronic mail: attention Mary Cutler, lab_info@solartron.com. More details about the program appear in http:// www.physics.unc.edu/ $\sim$ macd/.

${ }^{41}$ J. R. Macdonald, J. Chem. Phys. 102, 6241 (1995).

${ }^{42}$ J. R. Macdonald, J. Non-Cryst. Solids 197, 83 (1996); ibid. 204, 309 (1996); and $G_{D}$ in Eq. (A2) should be $G_{C D}$, the present $G_{1}$ quantity.

${ }^{43}$ J. R. Macdonald, Phys. Lett. A 220, 351 (1996).

${ }^{44}$ J. R. Macdonald, J. Non-Cryst. Solids 210, 70 (1997).

${ }^{45}$ J. R. Macdonald, J. Non-Cryst. Solids 212, 95 (1997). The $\sigma_{0}$ in Eq. (12) should be eliminated.

${ }^{46}$ J. R. Macdonald, Braz. J. Phys. (to be published).

${ }^{47}$ J. R. Macdonald, J. Appl. Phys. 82, 3962 (1997).

${ }^{48}$ J. R. Macdonald, in Impedance Spectroscopy_Emphasizing Solid Materials and Systems (Wiley-Interscience, New York, 1987).

${ }^{49}$ J. R. Macdonald, J. Appl. Phys. 58, 1955 (1985).

${ }^{50}$ P. B. Macedo, C. T. Moynihan, and R. Bose, Phys. Chem. Glasses 13, 171 (1972); and C. T. Moynihan, L. P. Boesch, and N. L. Laberge, ibid. 14, 122 (1973).

${ }^{51}$ C. Cramer, K. Funke, and T. Saatkamp, Philos. Mag. B 71, 701 (1995); C. Cramer, K. Funke, M. Buscher, A. Happe, T. Saatkamp, and D. Wilmer, ibid. 71, 713 (1995).

${ }^{52}$ K. L. Ngai (private communication, 1998).

${ }^{53}$ R. Kohlrausch, Pogg. Ann. Phys. Chem. 91, 179 (1854); G. Williams and D. C. Watts, Trans. Faraday Soc. 66, 80 (1970); G. Williams, D. C. Watts, S. B. Dev, and A. M. North, ibid. 67, 1323 (1971).

${ }^{54}$ F. Stickel, E. W. Fischer, and R. Richert, J. Chem. Phys. 102, 6251 (1995).

${ }^{55}$ D. W. Davidson and R. H. Cole, J. Chem. Phys. 39, 1417 (1961).

${ }^{56}$ J. R. Macdonald, Solid State Ionics 25, 271 (1987).

${ }^{57} \mathrm{H}$. Frohlich, Theory of Dielectrics (Oxford University Press, London, 1949).

${ }^{58}$ J. R. Macdonald, Phys. Rev. B 49, 9428 (1994-II).

${ }^{59}$ K. Funke, Prog. Solid State Chem. 22, 111 (1995).

${ }^{60}$ J. R. Macdonald, J. Appl. Phys. 34, 538 (1963).

${ }^{61}$ K. L. Ngai, A. K. Rajagopal, R. W. Rendell, and S. Teitler, Phys. Rev. B 28, 6073 (1983).

${ }^{62}$ J. R. Macdonald, J. Appl. Phys. 82, 1476 (1997).

${ }^{63}$ E. Helfand, J. Chem. Phys. 78, 1931 (1983); E. W. Montroll and J. T. Bendler, J. Stat. Phys. 34, 129 (1984). 